ThE ASTrophysiCAL Journal, 438:997-1012, 1995 January 10

(c) 1995. The American Astronomical Society. All rights reserved. Printed in U.S.A.

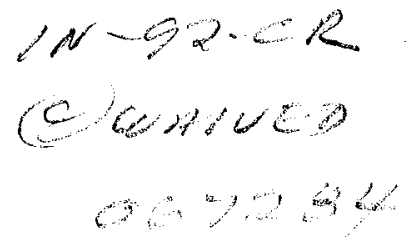
$6,2,46$

NASA/CR-95- 207165

\title{
CHROMOSPHERIC EVAPORATION AND DECIMETRIC RADIO EMISSION IN SOLAR FLARES
}

\author{
Markus J. Aschwanden \\ University of Maryland, Astronomy Department, College Park, MD 20742. E-mail: markus@astro.umd.edu \\ AND \\ ARNOLD O. BENZ \\ Institute of Astronomy, Federal Institute of Technology (ETH), CH-8092 Zurich, Switzerland \\ Received 1994 February 3; accepted 1994 July 13
}

\begin{abstract}
We have discovered decimetric signatures of the chromospheric evaporation process. Evidence for the radio detection of chromospheric evaporation is based on the radio-inferred values of (1) the electron density, (2) the propagation speed, and (3) the timing, which are found to be in good agreement with statistical values inferred from the blueshifted $\mathrm{Ca}$ XIX soft X-ray line. The physical basis of our model is that free-free absorption of plasma emission is strongly modified by the steep density gradient and the large temperature increase in the upflowing flare plasma. The steplike density increase at the chromospheric evaporation front causes a local discontinuity in the plasma frequency, manifested as almost infinite drift rate in decimetric type III bursts. The large temperature increase of the upflowing plasma considerably reduces the local free-free opacity (due to the $T^{-3 / 2}$ dependence) and thus enhances the brightness of radio bursts emitted at the local plasma frequency near the chromospheric evaporation front, while a high-frequency cutoff is expected in the high-density regions behind the front, which can be used to infer the velocity of the upflowing plasma. From model calculations we find strong evidence that decimetric bursts with a slowly drifting high-frequency cutoff are produced by fundamental plasma emission, contrary to the widespread belief that decimetric bursts are preferentially emitted at the harmonic plasma level.

We analyzed 21 flare episodes from 1991-1993 for which broadband (100-3000 MHz) radio dynamic spectra from Phoenix, hard X-ray data from BATSE/CGRO, and soft X-ray data from GOES were available. We detected slowly drifting high-frequency cutoffs between 1.1 and $3.0 \mathrm{GHz}$, with drift rates of $-41 \pm 32$ $\mathrm{MHz} \mathrm{s}^{-1}$, extending over time intervals of $24 \pm 23 \mathrm{~s}$. Developing a density model for type III-emitting flare loops based on the statistically observed drift rate of type III bursts by Alvarez \& Haddock (1973), we infer velocities of up to $360 \mathrm{~km} \mathrm{~s}^{-1}$ for the upflowing plasma, with an average of $v_{\mathrm{CE}}=236 \pm 130 \mathrm{~km} \mathrm{~s}$ for episodes with $5-15 \mathrm{~s}$ duration. The mean electron density of the upflowing plasma is $n_{e}=5.2( \pm 3.1) \times 10^{10}$ $\mathrm{cm}^{-3}$ when it is first detected in radio, at coronal altitudes of $h_{0}=9.2 \pm 2.3 \mathrm{Mm}$.
\end{abstract}

Subject headings: Sun: chromosphere - Sun: flares - Sun: radio radiation

\section{INTRODUCTION}

In the early phase of a solar flare, the plasma in a flare loop displays dynamic processes such as turbulent motion (with velocities exceeding $100 \mathrm{~km} \mathrm{~s}^{-1}$ ) and high-speed plasma upflows (with bulk velocities of $300-400 \mathrm{~km} \mathrm{~s}^{-1}$ ), as inferred from the line broadening and blueshift of soft X-ray (SXR) lines in Ca XIX and Fe XXV (Antonucci et al. 1982; Antonucci, Gabriel, \& Dennis 1984). The upward motion of the flare plasma in the early impulsive phase is generally referred to as "chromospheric evaporation" (Sturrock 1973) and is believed to be a consequence of local heating of the chromosphere near the footpoints of flare loops, produced either by collisions from precipitating electrons (Canfield et al. 1980) or by heat conduction (Antiochos \& Sturrock 1978). Chromospheric evaporation is considered to be the main mechanism for transporting the hot SXR-emitting flare plasma to coronal levels (Antonucci et al. 1984). Reviews on this subject can be found in Doschek et al. (1986), Antonucci (1989), Doschek (1990), and Antonucci et al. (1994).

The chromospheric evaporation process has thus far been studied chiefly in SXRs and $H \alpha$. In this paper we address for the first time observational evidence at radio wavelengths. The basic idea is that the local disturbance of the electron density and temperature, introduced by the upflowing chromospheric plasma, is detectable from radio bursts emitted at the local plasma frequency. Plasma emission produced by electron beams has been observed in the lower corona up to a frequency of $8.4 \mathrm{GHz}$ (Benz et al. 1992). The detection of plasma emission at such high frequencies requires overdense flux tubes, so that plasma emission can escape in a direction perpendicular to the flux-tube axis, where the density scale height is much shorter than in a homogeneous corona, and thus, free-free absorption is substantially reduced. In the event of chromospheric evaporation, we expect that the upflowing plasma surrounds overdense flux tubes and seals off escape routes for plasma emission, because the additional plasma material, if sufficiently dense, makes the escape routes optically thick owing to freefree absorption. Since the evaporating plasma propagates upward with a bulk speed of $\approx 300 \mathrm{~km} \mathrm{~s}^{-1}$, it is expected to produce a slowly drifting high-frequency cutoff for plasma emission. This high-frequency cutoff is thought to apply to any kind of plasma emission originating in "evaporating" flare loops, e.g., to type III bursts excited by precipitating electron beams. The drift rate of this high-frequency cutoff for plasma 
TABLE 1

Parameters of Plasma During Chromospheric Evaporation

\begin{tabular}{ccccc}
\hline \hline & $\begin{array}{c}\text { Velocity } \\
v_{\mathrm{CE}} \\
\left(\mathrm{km} \mathrm{s}^{-1}\right)\end{array}$ & $\begin{array}{c}\text { Temperature } \\
T_{e} \\
(\mathrm{MK})\end{array}$ & $\begin{array}{c}\text { Density } \\
10^{11} \mathrm{~cm}^{-3}\end{array}$ & $\begin{array}{c}\text { Plasma Frequency } \\
v_{p} \\
{[\mathrm{GHz}]}\end{array}$ \\
\hline Flare start ..... & $\ldots$ & $\ldots$ & $0.56-0.96$ & $2.1-2.8$ \\
Flare end $\ldots \ldots \ldots$ & $\ldots$ & $\ldots$ & $2.0-3.5$ & $4.0-5.3$ \\
Average $\ldots \ldots \ldots$. & $270 \pm 90$ & $16.5 \pm 2.4$ & $0.8-1.5$ & $2.5-3.5$ \\
\hline
\end{tabular}

emission is estimated to be

$$
\left(\frac{d v}{d t}\right)_{\mathrm{CE}}=\frac{\partial v}{\partial h} \frac{\partial h}{\partial t} \approx-\frac{v}{\lambda} v_{\mathrm{CE}} \cos \vartheta
$$

where $v$ is the observed frequency (assumed to be at the fundamental plasma frequency), $\lambda$ the local density scale height, $h$ the altitude, $v_{\mathrm{CE}}$ the velocity of the chromospheric evaporation front, and $\vartheta$ the propagation angle with respect to the vertical. Typical physical parameters of the chromospheric evaporating plasma are listed in Table 1 (from Antonucci et al. 1984). Based on these electron densities, we calculate that the plasma frequency of the upflowing plasma varies in the range of $v_{p}=$ 2.5-3.5 GHz and can be as low as $2.1 \mathrm{GHz}$ at the start of the flare. For an upward moving $(\vartheta=0)$ evaporation front, a velocity of $v_{\mathrm{CE}} \approx 270 \mathrm{~km} \mathrm{~s}^{-1}$, a scale height range of $\lambda=5-66$ Mm (inferred from decimetric type III bursts; Aschwanden \& Benz 1986), and a frequency of $v=2 \mathrm{GHz}$, we estimate a drift rate of $(d v / d t)_{\mathrm{CE}}=-8$ to $-108 \mathrm{MHz} \mathrm{s}^{-1}$ for the highfrequency cutoff. From this consideration we expect slowly drifting high-frequency cutoffs at decimetric frequencies for various radio bursts that are related to plasma emission, preferentially at the start of the impulsive phase of flares.

This paper is dedicated to testing these theoretical predictions and to exploring the effects of chromospheric evaporation at decimetric wavelengths. We search for evidence of radio signatures produced by chromospheric evaporation by using decimetric observations from Phoenix, which has been recently equipped with broadband receivers in the extended frequency range of $1-3 \mathrm{GHz}$. A representative selection of radio bursts observed in this little-explored frequency range can be found in Isliker \& Benz (1994). A substantial number of those radio bursts show slowly drifting high-frequency cutoffs at frequencies $\gtrsim 1 \mathrm{GHz}$, as expected from the considerations above.

In $\S 2$ we develop some theoretical tools to analyze decimetric signatures of chromospheric evaporation, in $\S 3$ we analyze flare observations at decimetric wavelengths and in $X$-rays, in $\S 4$ we discuss the results, and in $\S 5$ we summarize the findings and conclusions.

\section{THEORY}

First we need to develop some theoretical tools to perform a quantitative analysis of the data and to establish evidence for a high-frequency cutoff of plasma emission produced by the chromospheric evaporation process. In the following we outline a relevant flare scenario $(\$ 2.1)$, we derive a suitable density model for flare loops $(\S 2.2)$, we determine the velocity of chromospheric evaporation fronts $(\S 2.3)$, and perform numerical calculations of the free-free absorption for plasma emission originating in the environment of a chromospheric evaporating plasma $(\S 2.4)$.

\subsection{Flare Scenario}

A general scenario for the chromospheric evaporation process is depicted in Figure 1, where the typical time evolution of radio, hard X-ray (HXR), and SXR emission during different flare phases are outlined. Assuming that the primary energy release site where particle acceleration occurs is located in the corona, electron beams can be observed traveling both upward (as metric type III bursts) and downward (as decimetric reverse-slope [RS] drifting bursts) during the impulsive flare phase and sometimes in the preflare phase before HXR emission is detectable (Fig. 1, top). At about the onset of HXR emission, a chromospheric evaporation front starts to rise from the footpoints of the flare loops, characterized by a high temperature and a steep density gradient at the front. While the evaporation front ascends in the flare loops, it will intercept with downward-propagating electron beams (denoted RS in Fig. 1) in the flare loops. At the interface between the ascending evaporation front and the cooler flare loop, the free-free opacity will be abruptly reduced due to the higher temperature of the upflowing plasma, while the opacity increases again behind the evaporation front due to the increasing density. Thus, decimetric type III bursts intercepting the evaporation front will be observed at a higher brightness temperature at the interface of the evaporation front due to the reduced free-free opacity, and will exhibit a much higher frequency-drift rate due to the locally steep density gradient at the evaporation front. After the (RS) type III bursts have crossed the evaporation front, plasma emission will be completely absorbed due to the higher density and increased path length in the high-density medium, which is observed as a high-frequency cutoff. This cutoff drifts slowly toward lower frequencies (Fig. 1, top) as the evaporation front reaches higher altitudes (Fig. 1, bottom), because the local plasma frequency decreases with height. SXR emission is produced in the volume of the upflowing hot plasma, leading to a monotonic increase of the SXR emission measure during the impulsive phase (Fig. 1, middle). The trapped particles in the flare loop undergo kinetic plasma instabilities, occasionally resulting in coherent radio emission (e.g., observed in the form of decimetric radio pulsations). If the coherent radio emission is produced by waves that are related to the plasma frequency, e.g., harmonic plasma waves or upper hybrid waves, we expect that they are subject to the same high-frequency cutoff as the decimetric type III bursts (radio emission from the trap is marked with a hatched area in Fig. 1, top). The boundaries of the trap at the mirrorpoints are then pushed together from the ascending chromospheric plasma (Fig. 1, bottom). Eventually, the evaporated chromospheric material reaches the apex of the flare loops or reaches pressure equilibrium, and the high-frequency cutoff for plasma emission will disappear.

\subsection{Density Model for Type III-Emitting Flare Loops}

The coronal electron density and temperature in flaring active regions are highly inhomogeneous. Thus, there is no standard density model that characterizes flare loops and the quiet corona equally well. Existing density models of the corona are based on scattered white light for the quiet corona (e.g., Baumbach-Allen model, Newkirk model), with an arbitrary adjustment factor of 5-10 for active regions. The models assume hydrostatic equilibrium, either for an isothermal atmosphere (barometric model) or including the temperature gradient in the upper transition region by constraints of the 


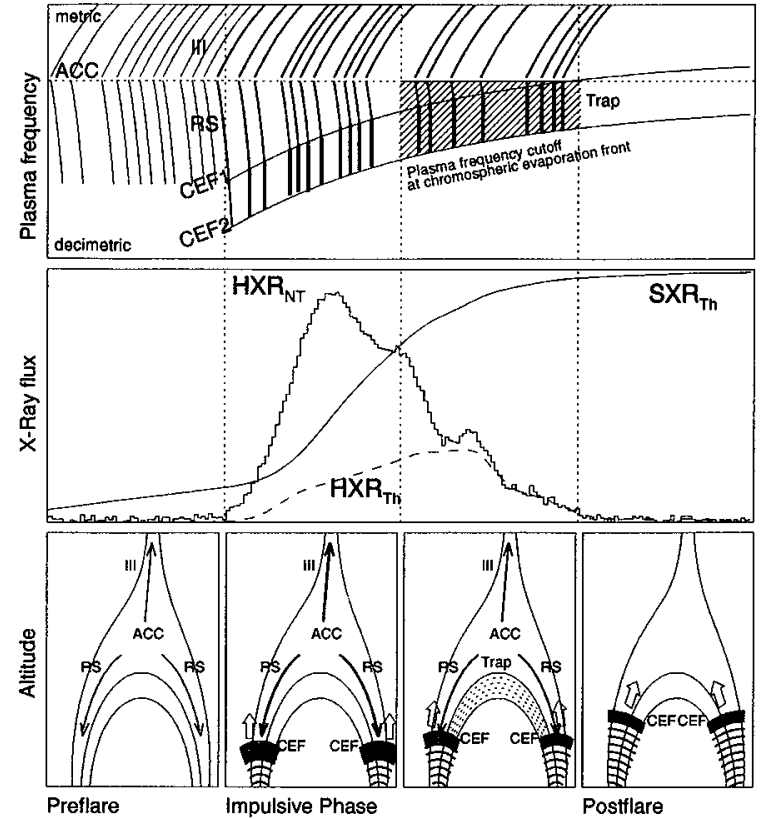

Fig. 1.-Flare scenario illustrating the evolution of chromospheric evaporation (CEF), nonthermal hard X-ray emission $\left(\mathrm{HXR}_{\mathrm{NT}}\right)$, thermal hard X-ray emission ( $\mathrm{HXR}_{\mathrm{Th}}$ ), soft X-ray emission ( $\mathrm{SXR}_{\mathrm{Th}}$ ), and radio emission (type III bursts, reverse-slope [RS] bursts, and plasma emission from a trap). ACC denotes the coronal acceleration region. In the radio dynamic spectrum (top) a slowly drifting plasma frequency cutoff is expected owing to the density enhancement conveyed by the chromospheric evaporation front; CEF1 and CEF2 mark the plasma frequencies corresponding to the low-density (CEF1) and high-density edge (CEF2) at the evaporation front. The density of the upflowing chromospheric plasma is indicated with contour lines (bottom), marking a steep gradient at the evaporation front that corresponds to the jump of the plasma frequency from CEF1 to CEF2 (top). This regime CEF1-CEF2 is also the frequency range where the opacity changes for plasma emission are most dramatic, and where the drift rate of type III bursts becomes almost infinite.

conductive flux (Dulk et al. 1977). The Baumbach-Allen model and the barometric model for an isothermal corona with a temperature of $1.5 \mathrm{MK}$ are shown in Figure 2. These models are not appropriate for type III-emitting flare loops for two reasons: (1) they represent lower limits on the electron density in type III-emitting flare loops, because the escape condition for plasma emission (to overcome free-free absorption) dictates overdense loops (e.g., by a factor of $\approx 18$ in Benz et al. 1992), and (2) flare loops are subject to substantial heating and mass flow, and are therefore not in hydrodynamic equilibrium.

Here we are interested in estimating the electron density in flare loops where chromospheric evaporation and plasma emission from injected electron beams occurs. We construct an empirical density model $n_{e}(h)$ by using the statistically observed drift rate of type III bursts according to Alvarez \& Haddock (1973),

$$
\frac{\partial v}{\partial t}=-A v^{\alpha}
$$

which follows a power-law function of the frequency, $A$ and $\alpha$ being constants. At large coronal heights, hydrostatic equilibrium is considered to be a good approximation and a barometric density model can be justified with a density scale height corresponding to the coronal temperature. Thus, we combine the two models by assuming a power-law function in the lower

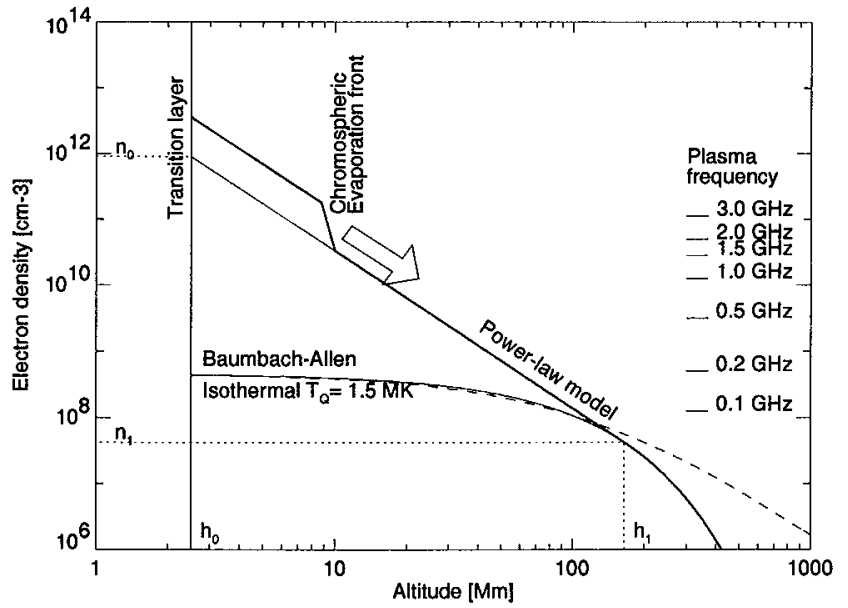

FIG. 2.-Density model (thick line) for type III-emitting (overdense) flux tubes, consisting of a barometric component for $h>h_{1}$, a power-law component for $h<h_{1}$, and a (time-dependent) enhanced density component associated with the upflowing chromospheric plasma (indicated here at a height of $\lesssim 10 \mathrm{Mm}$ ). The Baumbach-Allen model (dashed line) matches the barometric model (thin line) for heights $h \lesssim h_{1}$. The plasma frequency scale is indicated on the right.

corona and an exponential form in the upper corona, i.e.,

$$
n_{e}(h)= \begin{cases}n_{1}\left(h / h_{1}\right)^{-p} & h<h_{1}, \\ n_{Q} \exp (-h / \lambda) & h>h_{1},\end{cases}
$$

with a transition at height $h_{1}$, specified by the requirement of a smooth transition between the two regimes. The derivation of the numerical constants $n_{1}, h_{1}, n_{Q}$ and $\lambda$ is given in Appendix A. This density model for flare loops is shown in Figure 2. Since decimetric type III bursts occur preferentially in closed (flare) loops (Aschwanden, Benz, \& Montello 1994), while metric type III bursts generally originate from electron beams propagating along open field lines, our density model represents a gradual transition from closed to open magnetic structures with height. Nevertheless, it is intended to provide a representative density function for type III-emitting structures in a statistical sense. It provides the basis for measuring speeds of the chromospheric evaporation front with radio methods.

\subsection{The Velocity of the Chromospheric Evaporation Front}

The drift rate of a type III burst is proportional to the exciter speed for a given frequency $v$ (see eq. [A6] in Appendix A). This proportionality is equally true for any propagating agent that affects plasma emission. In particular, we can relate the slowly drifting high-frequency cutoff of plasma emission to the velocity of the chromospheric evaporation front in the same way. Since both the type III bursts and the plasma frequency cutoff occur in the same flare loop described by our density model (eq. [3]), we can apply the drift rate relation (A6) to both exciters. Hence, the two relations $(\partial v / \partial t)_{\mathrm{III}} \propto v_{\mathrm{III}}$ and $(\partial v / \partial t)_{\mathrm{CE}} \propto v_{\mathrm{CE}}$ have the same proportionality constant (in eq. [A6]), and the velocity $v_{\mathrm{CE}}$ of a chromospheric evaporation front can be found from

$$
v_{\mathrm{CE}}=v_{\mathrm{III}} \frac{(\partial v / \partial t)_{\mathrm{CE}}}{(\partial v / \partial t)_{\mathrm{III}}}
$$

The drift rate of type III bursts, $(\partial v / \partial t)_{\text {II }}$ can be inserted from equation (2) of Alvarez \& Haddock (1973). A representative 
TABLE 2

TYPE III EXCITER SPEedS

\begin{tabular}{clcc}
\hline \hline $\begin{array}{c}\text { Velocity } \\
v_{\text {III }} / \mathrm{c}\end{array}$ & \multicolumn{1}{c}{$\begin{array}{c}\text { Frequency } \\
\text { Range } \\
(\mathrm{MHz})\end{array}$} & $\begin{array}{c}\text { Number of } \\
\text { Analyzed } \\
\text { Events }\end{array}$ & Reference \\
\hline $0.45 \ldots \ldots \ldots \ldots$. & $45-60 \mathrm{MHz}$ & 8 & 1 \\
$0.33 \ldots \ldots \ldots \ldots$ & $7-220 \mathrm{MHz}$ & 50 & 2 \\
$0.15 \pm 0.03 \ldots \ldots$ & $160-320 \mathrm{MHz}$ & 12 & 3 \\
$0.16 \pm 0.05 \ldots \ldots$ & $56 \mathrm{kHz}$ & 3 & 4 \\
$0.14 \pm 0.04 \ldots \ldots$ & $1 \mathrm{MHz}$ & 28 & 5 \\
$0.18 \pm 0.04 \ldots \ldots$ & $1.446 \mathrm{GHz}$ & 1 & 6 \\
$0.15 \pm 0.02 \ldots \ldots$ & $250-850 \mathrm{MHz}$ & 1 & 7 \\
$0.30 \pm 0.10 \ldots \ldots$ & $560-1240 \mathrm{MHz}$ & 1 & 7 \\
\hline $0.15 \pm 0.04 \ldots \ldots$ & $56 \mathrm{kHz}-1.5 \mathrm{GHz}$ & $\ldots$ & 8 \\
\hline
\end{tabular}

REFERENCES - (1) Wild, Sheridan, \& Neylan 1959; (2) Stewart 1965 (3) Fokker 1970 ; (4) Lin et al. 1981, 1986; (5) Dulk et al. 1987; (6) Aschwanden et al. 1992; (7) Aschwanden 1993; (8) Weighted average (References after 1970).

value of the type III exciter speed is $v_{\mathrm{III}} / c=0.15 \pm 0.04$ (see average value in Table 2, a discussion in the context of our model is given in Appendix B). Figure 3 shows the drifting plasma frequency cutoff in a radio dynamic spectrum, calculated for chromospheric evaporation fronts with upward velocities of $v_{\mathrm{CE}}=100,200$, and $300 \mathrm{~km} \mathrm{~s}^{-1}$. Note that the detection of a plasma frequency cutoff at $<3 \mathrm{GHz}$ is delayed by more than $20 \mathrm{~s}$ with respect to the star of a chromospheric evaporation, for propagation speeds of $v_{\mathrm{CE}} \leq 300 \mathrm{~km}$.

\subsection{The High-Frequency Cutoff of Plasma Emission}

We calculate the radio spectrum of plasma emission in the environment of a chromospheric evaporation front, with particular emphasis on the high-frequency cutoff. The free-free absorption coefficient in typical coronal conditions can be approximated (Dulk 1985) by

$$
\kappa_{\mathrm{ff}} \approx 0.2 n_{e}^{2} T^{-3 / 2} v^{-2} \mathrm{~cm}^{-1}
$$

In the case of plasma emission the observed frequency $v$ can be expressed as a function of the local density $n_{e}$ by the plasma

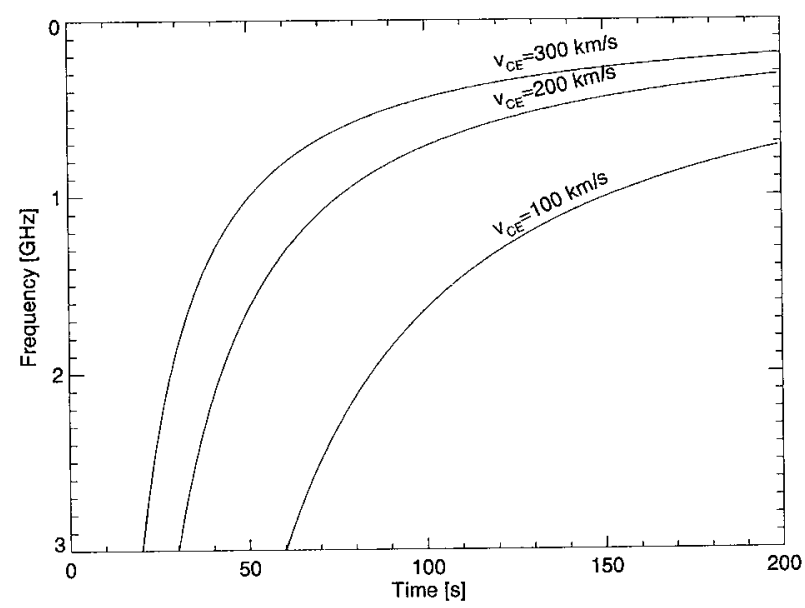

FIG. 3-Evolution of the plasma frequency cutoff calculated for an upflowing chromospheric evaporation front with a constant velocity of $v_{\mathrm{CE}}=$ 100,200 , or $300 \mathrm{~km} \mathrm{~s}^{-1}$, according to the density model illustrated in Fig. 2. frequency relation (A3). The resulting optical depth along the path $r$ is then

$$
\tau_{\mathrm{ff}}(r)=\int_{r}^{\infty} \kappa_{\mathrm{ff}} d r=2.5 \times 10^{-9} \frac{1}{s^{2}} \int_{r}^{\infty} n_{e}(r) T(r)^{-3 / 2} d r .
$$

The observed radio brightness is reduced by the absorption factor $e^{-\tau_{f f}}$. If the intrinsic brightness temperature of the radio source is characterized by the spectrum $T_{B 0}(v)$, the observed spectrum will be

$$
T_{B}(v)=T_{B 0}(v) \exp ^{-\tau_{f f}(r[v])},
$$

where the frequency dependence of the location $r(v)$ is given by the plasma frequency relation $v(r)=s 8980 n_{e}(r)^{-1 / 2}$ and the density model $n_{e}(r)$.

In order to study the effects of a chromospheric evaporation front on the observed radio spectrum of plasma emission, we perform numerical calculations of the radio brightness temperature $T_{B}(v)$. We consider the situation of a vertical loop embedded in the ascending chromospheric evaporation plasma. The electron density in the overdense loop is specified by our density model (eq. [3]) in the vertical direction, and by a Gaussian distribution with half-width $d$ in the horizontal direction. The chromospheric evaporation plasma is approximated by a horizontally stratified structure, which has a density that is enhanced by a factor of $q_{\mathrm{CE}}=4$ relative to the embedded flux tube (suggested by the average density variation measured from Ca XIX during a flare, see Table 1). The front of the upflowing plasma moves with a velocity of $v_{\mathrm{CE}}$ and the interface is located at height $h_{\mathrm{CE}}=v_{\mathrm{CE}} t$ for times $t>0$. The twodimensional distribution of the electron density,

$$
n_{e}(h, x, t)= \begin{cases}n_{Q} \exp \left(-\frac{h}{\lambda}\right) \exp ^{-(x / d)^{2}} & h>h_{1}, \\ n_{Q}\left(\frac{e}{p} \frac{h}{\lambda}\right)^{-p} \exp ^{-(x / d)^{2}} & h_{\mathrm{CE}}(t)<h<h_{1}, \\ n_{Q} q_{\mathrm{CE}}\left(\frac{e}{p} \frac{h}{\lambda}\right)^{-p} & h<h_{\mathrm{CE}}(t),\end{cases}
$$

is shown in the form of a contourplot in Figure 4 (left). The structure of the magnetic field that defines the vertical flux tube is assumed to be unaffected by the upflowing plasma. For the temperature distribution we simply assume a coronal temperature of $T_{C}=1.5 \mathrm{MK}$ for the overdense loop and a hotter temperature of $T_{\mathrm{CE}}=15 \mathrm{MK}$ for the chromospheric evaporation front,

$$
T(h, x, t)= \begin{cases}T_{C} & h>h_{\mathrm{CE}}(t), \\ T_{\mathrm{CE}} & h<h_{\mathrm{CE}}(t) .\end{cases}
$$

For a given line-of-sight with aspect angle $\vartheta$ to the vertical, the coordinates are $x=r \sin (\vartheta)$ and $y=h+r \cos (\vartheta)$, for a ray starting at height $h$ at the center $(x=0)$ of the cylindrical flux tube. The radio brightness spectrum $T_{B}[v(h)]$ originating from the center of the overdense flux tube can then be calculated in a straightforward manner by numerical integration along a selected line of sight using the two-dimensional distributions $n_{e}(h, x, t)$ and $T(h, x, t)$ (eqs. [8] and [9]) for a given time $t$.

The resulting radio spectrum $T_{B}(v) / T_{B 0}(v)$ is shown in Figure 4 (bottom right) for a flux-tube radius of $d=2 \mathrm{Mm}$, for a height of $h_{\mathrm{CE}}=20 \mathrm{Mm}$ of the chromospheric evaporation front, and for an aspect angle of $\vartheta=45^{\circ}$. Note that the spectrum is normalized by the intrinsic brightness temperature $T_{B 0}$ that corresponds to the spectrum without the effect of free-free 

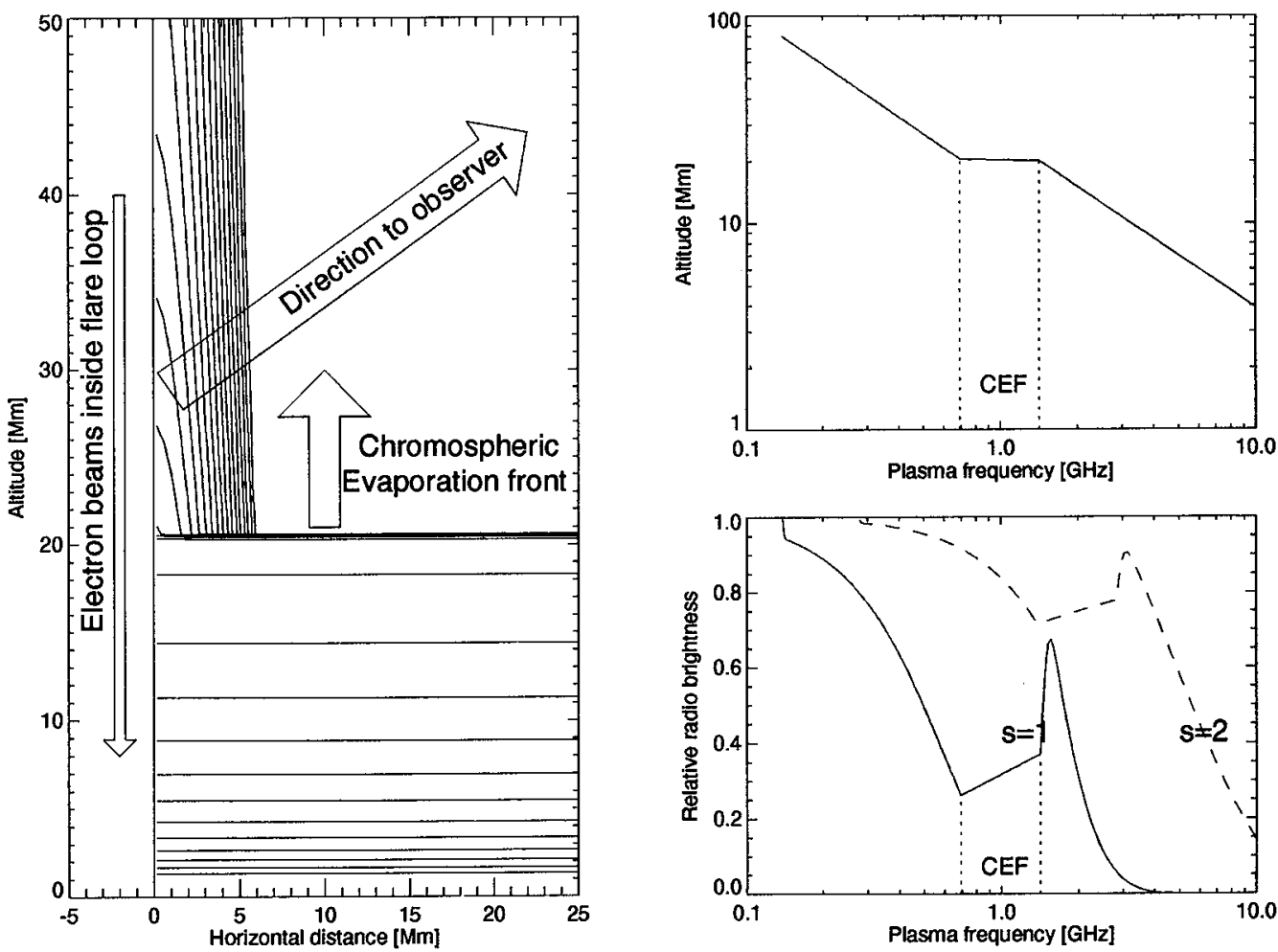

FIG. 4.-Two-dimensional density model with a vertical flux tube (with FWHM of $2 \mathrm{Mm}$ ) and a horizontally stratified chromospheric evaporation front (CEF) at a height of $\leq 20 \mathrm{Mm}$. The two-dimensional density distribution is shown (left) with logarithmic contour levels (in steps of 0.25 ). The vertical density distribution is identical with the density model in Fig. 2, except that here the height of the CEF is $20 \mathrm{Mm}$. The dependence of the plasma frequency vs. height is shown (top right). The radio brightness spectrum $T_{B}(v) / T_{B 0}(v)$ of fundamental $(s=1)$ and harmonic $(s=2)$ plasma emission excited by an electron beam propagating vertically along the overdense flux tube is calculated for an aspect angle of $45^{\circ}$ (bottom right).

absorption (as defined in eq. [7]). The effect of the chromospheric evaporation front on the radio spectrum can clearly be seen in this example: (1) since the density structure at the evaporation front was assumed to be a step function with an enhancement factor of $q_{\mathrm{CE}}=4$, the plasma frequency jumps by a factor of 2 (from $v_{\mathrm{CE} 1}=0.7 \mathrm{GHz}$ to $v_{\mathrm{CE} 2}=1.4 \mathrm{GHz}$ ), while the opacity is almost constant over this frequency range; (2) above the frequency $v_{\mathrm{CE} 2}$, the brightness first increases, with a peak at $\approx 1.7 \mathrm{GHz}$, because the opacity (proportional to $n_{e} T^{-3 / 2}$ ) is strongly reduced due to the higher temperature $\left(T_{\mathrm{CE}} / T_{C}=10\right)$; (3) above $v \approx 1.7 \mathrm{GHz}$ the radio spectrum rapidly falls off because the opacity gradually increases due to the increasing path length $\operatorname{dr}(h)=\left(h_{\mathrm{CE}}-h\right) / \cos (9)$ in the highdensity region behind the chromospheric evaporation front $\left(h<h_{\mathrm{CE}}\right)$. The transit of the chromospheric evaporation front produces a relatively narrowband structure in the decimetric spectrum, with a bandwidth of $\Delta v \approx 0.6 \mathrm{GHz}$ at $v \approx 1.7 \mathrm{GHz}$ (i.e., $\Delta v / v \approx 0.35$ in this example). For harmonic plasma emission (dashed spectrum in Figure 4, bottom right), the effect of the hot evaporation front is much less dramatic and does not produce a strong change in the $1-3 \mathrm{GHz}$ frequency range of interest here. The high-frequency cutoff is relatively broad and extends to $\gtrsim 10 \mathrm{GHz}$. Flux tubes with very large diameters $(\gtrsim 50 \mathrm{Mm}$ ) would be required in order to produce a similar effect in the $\leq 3 \mathrm{GHz}$ range for harmonic plasma emission.

Time-dependent simulations for chromospheric evaporation fronts (i.e., $\left.h_{\mathrm{CE}}=v_{\mathrm{CE}} t\right)$ with different temperatures $\left(T_{\mathrm{CE}}=10\right.$ MK and $T_{\mathrm{CE}}=20 \mathrm{MK}$ ) are shown in Figure 5, assuming a constant velocity of the evaporating plasma $\left(v_{\mathrm{CE}}=300 \mathrm{~km}\right.$ $\mathrm{s}^{-1}$ ), illustrated for a periodic sequence of bursts that emit at the fundamental plasma frequency. The chromospheric evaporation front passes the $3 \mathrm{GHz}$ plasma level between 20 s and 36 $s$ and makes the flux tube optically transparent after this time, because of the higher temperature of the evaporating material. A new decimetric component becomes visible after $36 \mathrm{~s}$, whose peak and start frequencies drift slowly toward lower frequencies. For the $20 \mathrm{MK}$ hot chromospheric evaporation front the peak frequency is $2 \mathrm{GHz}$ about $60 \mathrm{~s}$ after start, and drifts toward $1 \mathrm{GHz}$ after $110 \mathrm{~s}$. The start frequency of this decimetric component drifts similarly toward lower frequencies, while the spectral cutoff at the high-frequency side is steeper for the cooler $\left(T_{\mathrm{CE}}=10 \mathrm{MK}\right)$ chromospheric material. According to the theoretical calculations above, the variation of the radio start frequency is merely a temperature effect: the free-free opacity is drastically reduced after the arrival of the hot upflowing plasma due to the $T^{-3 / 2}$ dependence in the free-free absorption coefficient (eq. [5]). Thus, the start frequency of radio bursts emitted at the plasma frequency bear an important potential for temperature diagnostics.

We investigate the dependence of the radio spectrum on various parameters in Figure 6 . The calculated radio spectrum $T_{B}(v) / T_{B 0}(v)$ is shown for fundamental $(s=1$; Fig. 6, left side $)$ and harmonic ( $s=2$; Fig. 6 , right side) plasma emission, for several values of the temperature of the upflowing plasma $\left(T_{\mathrm{CE}}=7.5,15,30\right.$, MK; Fig. 6, top row $)$, the aspect angle $\left(\vartheta=10^{\circ}, 45^{\circ}, 80^{\circ}\right.$; Fig. 6 , middle row $)$, and the flux-tube radius $(d=1,2,4 \mathrm{Mm}$; Fig. 6 , bottom row). From these computations we see that (1) the fundamental plasma emission has a steep high-frequency cutoff which is below $3 \mathrm{GHz}$ in most of the cases, while the harmonic plasma emission cutoff is more 

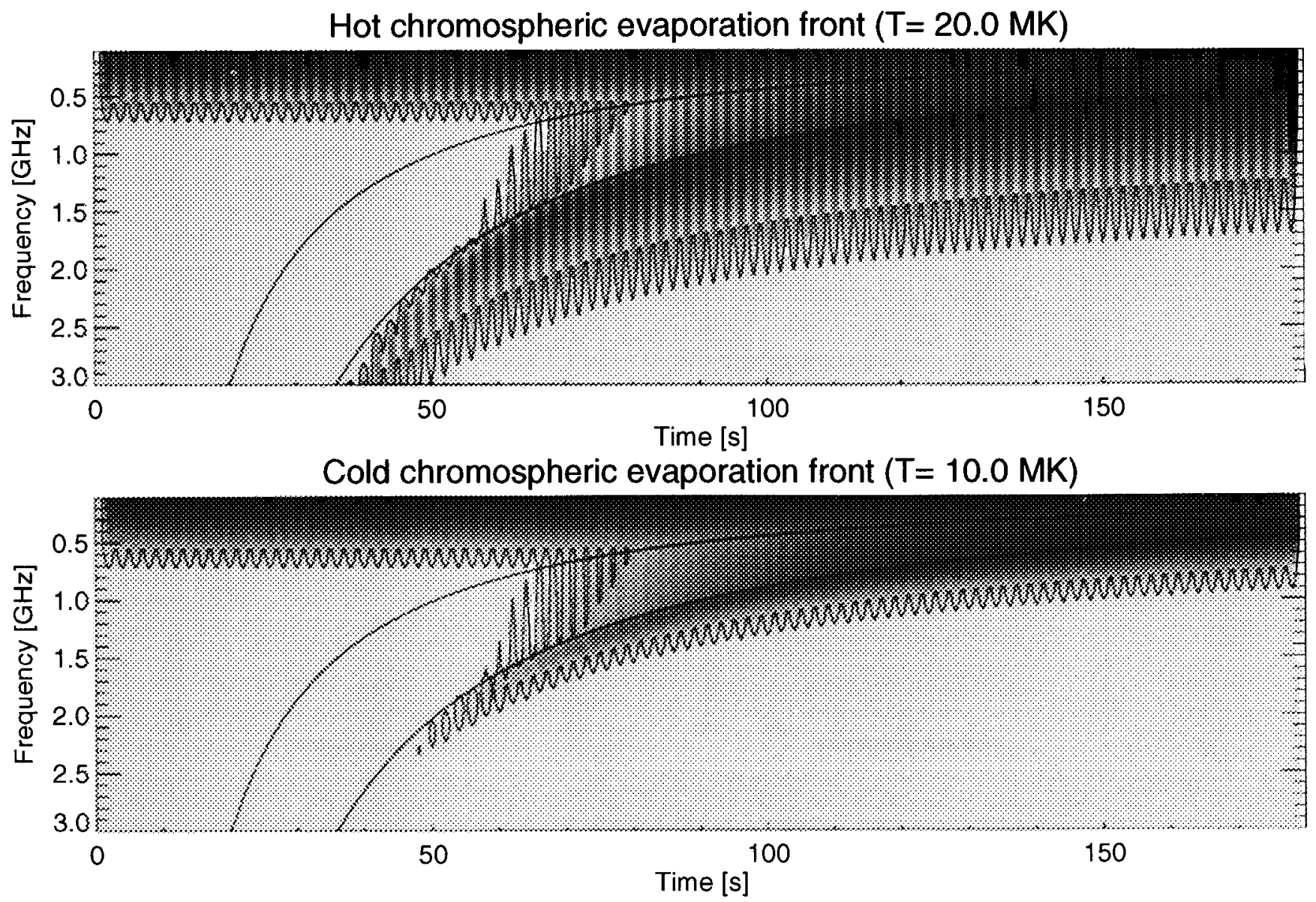

Fig. 5.- Simulated evolution of the radio dynamic spectrum during the first 3 minutes of a flare using the scenario described in the text. The radio spectrum is calculated for a periodic sequence of bursts emitting at the fundamental plasma frequency and propagating along the center of an overdense loop of radius $2 \mathrm{Mm}$. The curved trajectories mark the plasma frequency at the low- and high-density edges of a chromospheric evaporation front propagating with a velocity of $300 \mathrm{~km}$ $\mathrm{s}^{-1}$ upward in the corona, with a temperature of $20 \mathrm{MK}($ top $)$, or $10 \mathrm{MK}$ (bottom). The density enhancement in the evaporation front is assumed to be a factor of 4. Note the enhanced radio brightness and the high-frequency cutoff associated with the (slowly drifting) passage of the evaporation front.

smooth and is above $\approx 3 \mathrm{GHz} ;(2)$ a higher temperature of the upflowing plasma shifts the start frequency to higher values; (3) radio bursts at the solar $\operatorname{limb}\left(\vartheta \geq 80^{\circ}\right)$ have a steeper spectral cutoff and a lower cutoff frequency than do bursts from intermediate $\left(9 \approx 45^{\circ}\right)$ longitudes; (4) radio bursts from near the center of the solar disk are also subject to strong absorption because the column depth of free-free absorption along the vertical axis of the flux tube is large; (5) the flux-tube diameter essentially determines the suppression of plasma emission at the low-frequency side, while the high-frequency cutoff is not affected by the flux-tube diameter.

\section{OBSERVATIONS}

We analyze radio observations from the radio spectrometer Phoenix at Bleien, operated by ETH Zürich (Switzerland), HXR observations from the Compton Gamma-Ray Observatory (CGRO), and soft X-ray observations from the Geostationary Operational Environmental Satellites (GOES).

\subsection{Instruments and Data}

The frequency-agile broadband spectrometer Phoenix, which covers the frequency range of $0.1-3.0 \mathrm{GHz}$, was put into operation in 1989 June. Instrumental descriptions and first results are given in Benz et al. (1991) and Isliker \& Benz (1994). Since 1992 September, Phoenix has been operated mostly in a broadband mode with continuous coverage over the full frequency range of $0.1-3.0 \mathrm{GHz}$, using a time resolution of $0.1 \mathrm{~s}$. These broadband observations are the most useful ones for the purpose of our study. We analyzed 30 events with simultaneous detection of HXR emission by $C G R O$, plus an additional flare without $C G R O$ data. In at least 11 out of these 31 flares we found radio signatures of chromospheric evaporation, which is a lower limit, since not all events had optimum frequency and time coverage in radio.

Hard X-ray data from CGRO are obtained from the Burst and Transient Source Experiment (BATSE). We use BATSE background data (DISCLA data type), with $1.024 \mathrm{~s}$ time resolution, from the eight large-area detectors (LADs), with $2000 \mathrm{~cm}^{-3}$ detector area each, in the four energy channels of $25-50 \mathrm{keV}, 50-100 \mathrm{keV}, 100-300 \mathrm{keV}$, and $>300 \mathrm{keV}$. Instrumental descriptions of BATSE data can be found in Fishman et al. $(1989,1992)$. The BATSE data were provided by the Solar Data Analysis Center (SDAC) at NASA/Goddard Space Flight Center (GSFC).

SXR data were obtained from the NOAA-operated GOES satellites, with wavelength ranges of $0.5-4 \AA$ and $1-8 \AA$, corresponding to energy bands of $3-25 \mathrm{keV}$ and $1.5-12 \mathrm{keV}$. The 

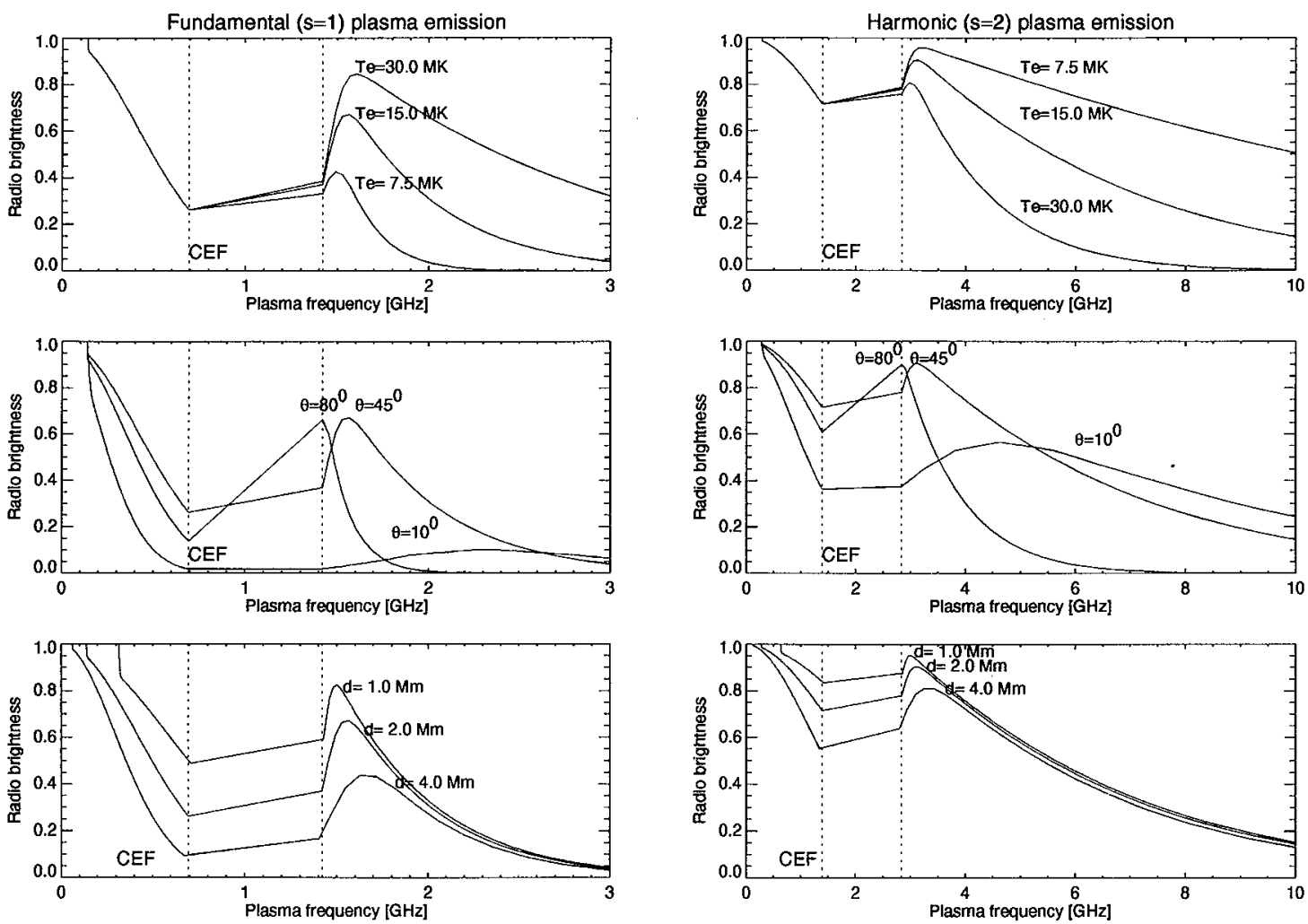

FIG. 6. - Calculated radio spectra in parameter space, for fundamental $\left(s=1\right.$, left) and harmonic $\left(s=2\right.$, right) plasma emission, and for different temperatures $\left(T_{e}\right.$, top row), aspect angles $\left(\vartheta\right.$, middle row), and flux tube diameters (d, bottom row). The unvaried parameters are the same as in Fig. $4: d=2 \mathrm{Mm}, h_{\mathrm{C}}=20 \mathrm{Mm}, T_{\mathrm{CF}}=15$ MK, $q_{\mathrm{EV}}=4, \vartheta=45^{\circ}$, and $\alpha=1.84$. Note that the frequency scales are linear but different for fundamental $(0.1-3.0 \mathrm{GHz})$ and harmonic $(0.1-10 \mathrm{GHz})$ plasma emission.

time resolution of the GOES data is $3 \mathrm{~s}$. The SXR data were provided through the GOES Archive at SDAC.

\subsection{Data Analysis}

In the analysis of the radio data, we focus on those flare episodes in which a high-frequency cutoff is apparent in the radio dynamic spectra of $0.1-3.0 \mathrm{GHz}$. We select time intervals with a continuous high-frequency cutoff, regardless of whether the enhanced emission below the cutoff frequency consists of continuum emission (e.g., type IV bursts) or of fast-drift bursts (e.g., type IIIdm bursts or decimetric broadband pulsations). The analysis presented here contains 21 episodes extracted from 11 different flares.

During a selected time interval $t_{i}, i=1, \ldots, n$ with $n$ datapoints (measured with a typical time resolution of $0.1 \mathrm{~s}$ ) we determine the cutoff frequencies $v_{i}, i=1, \ldots, n$ (or start frequency) from the highest frequency where the radio spectrum $F_{i}\left(v, t=t_{i}\right)$ exceeds a threshold of 3 times the rms noise level above the background. In the case of bursty emission, we sample the median values of the start frequency $v_{i}$ from larger time bins (with $\approx 1$ s duration). Almost all of the episodes show a high-frequency cutoff $v_{i}\left(t_{i}\right)$ that drifts toward lower frequencies, while drifts toward higher frequencies were found to be rare, and are omitted in the subsequent analysis.

We fit a theoretical dynamic spectrum $v(t)$ of an exciter moving with constant velocity $v$ toward a lower density region. Using the relation for the dynamic spectrum $v(t)$ derived for overdense loops (A5), which can be written as

$$
\frac{p}{e}\left(\frac{v}{s v_{Q}}\right)^{-(2 / p)}=\frac{h_{0}}{\lambda}+\frac{v}{\lambda}\left(t-t_{0}\right),
$$

we perform a linear regression fit with the variables $x_{i}, y_{i}$, defined by

$$
y_{i}=a_{0}+\left(a_{1} \cdot x_{i}\right)\left\{\begin{array}{l}
y_{i}=\frac{p}{e}\left(\frac{v_{i}}{s v_{Q}}\right)^{-(2 / p)}, \\
x_{i}=\left(t_{i}-t_{0}\right) \\
a_{0}=\frac{h_{0}}{\lambda} \\
a_{1}=\frac{v}{\lambda}
\end{array}\right.
$$

Thus, for each time interval with a high-frequency cutoff $v_{i}\left(t_{i}\right)$, we obtain from the linear regression coefficients $\left(a_{0}, a_{1}\right)$ the two constants $h_{0}=a_{0} \lambda$ and $v=a_{1} \lambda$, which are the altitude $h_{0}=$ $h\left(t=t_{0}\right)$ and the velocity $v$ of an exciter that propagates in an overdense flux tube, specified by our density model (eq. [3]) and the parameters given in equation (A9). The so derived values for $h_{0}$ and $v$ apply to fundamental $(s=1)$ plasma emission, according to the arguments given in Appendix B. For harmonic $(s=2)$ plasma emission, the values for $v$ and $h_{0}$ would be a factor of $s^{2 / p}=2^{2 / 2.38}=1.79$ higher. 


\subsection{Selected Flare Events}

In the following we describe three selected flares, which show the existence of a high-frequency cutoff for three different types of radio emission: for a type IV event (Fig. 7), a decimetric pulsation event (Fig. 8), and dm type III bursts (Fig. 9).

\subsubsection{Type IV Event}

Figure 7 shows the radio data from Phoenix and SXR data from GOES. Since there are no HXR data available for this flare, we show the time derivative of the SXR data as a proxy for the HXR data (see discussion of the Neupert effect in Dennis \& Zarro 1993). The first significant increase in the time derivative of the SXR flux (Fig. 7, bottom) occurs at 1200:50 UT. The start of the GOES M3.6 flare was reported at 1159 UT (Solar Geophysical Data), in coincidence with an $\mathrm{H} \alpha$ flare in NOAA Active Region 6718 at heliographic position S22E34. Enhanced decimetric radio emission was detected by the burst trigger of Phoenix at 1201:26 UT, which is the start of the digitally recorded radio portion shown in Figure 7 . This radio event was classified as type IV burst with fast-drifting fine structures and parallel-drifting bands ("zebra" patterns) in Isliker \& Benz (1994; see Fig. 26 therein).

The radio data show a persistent high-frequency cutoff throughout the event, which drifts slowly from $3 \mathrm{GHz}$ initially (36 s after flare start in SXR) to $2.5 \mathrm{GHz}$ (60 s after flare start). During this time interval there occur four episodes, of $1.2-4.5 \mathrm{~s}$ duration, showing a distinct high-frequency cutoff which exhibits a slow drift rate between -36 and $-90 \mathrm{MHz} \mathrm{s}^{-1}$ in the frequency range of $2.5-3.0 \mathrm{GHz}$. From these drift rates we infer with equation 4 upward-directed velocities between 60 and $153 \mathrm{~km} \mathrm{~s}^{-1}$. If we interpret the radio emission in terms of fundamental plasma emission, the observed frequency range corresponds to electron densities of $n_{e}=0.9-1.2 \times 10^{11} \mathrm{~cm}^{-3}$. These values appear to be remarkably close to the typical values inferred from the blueshift of the Ca XIX line at the onset
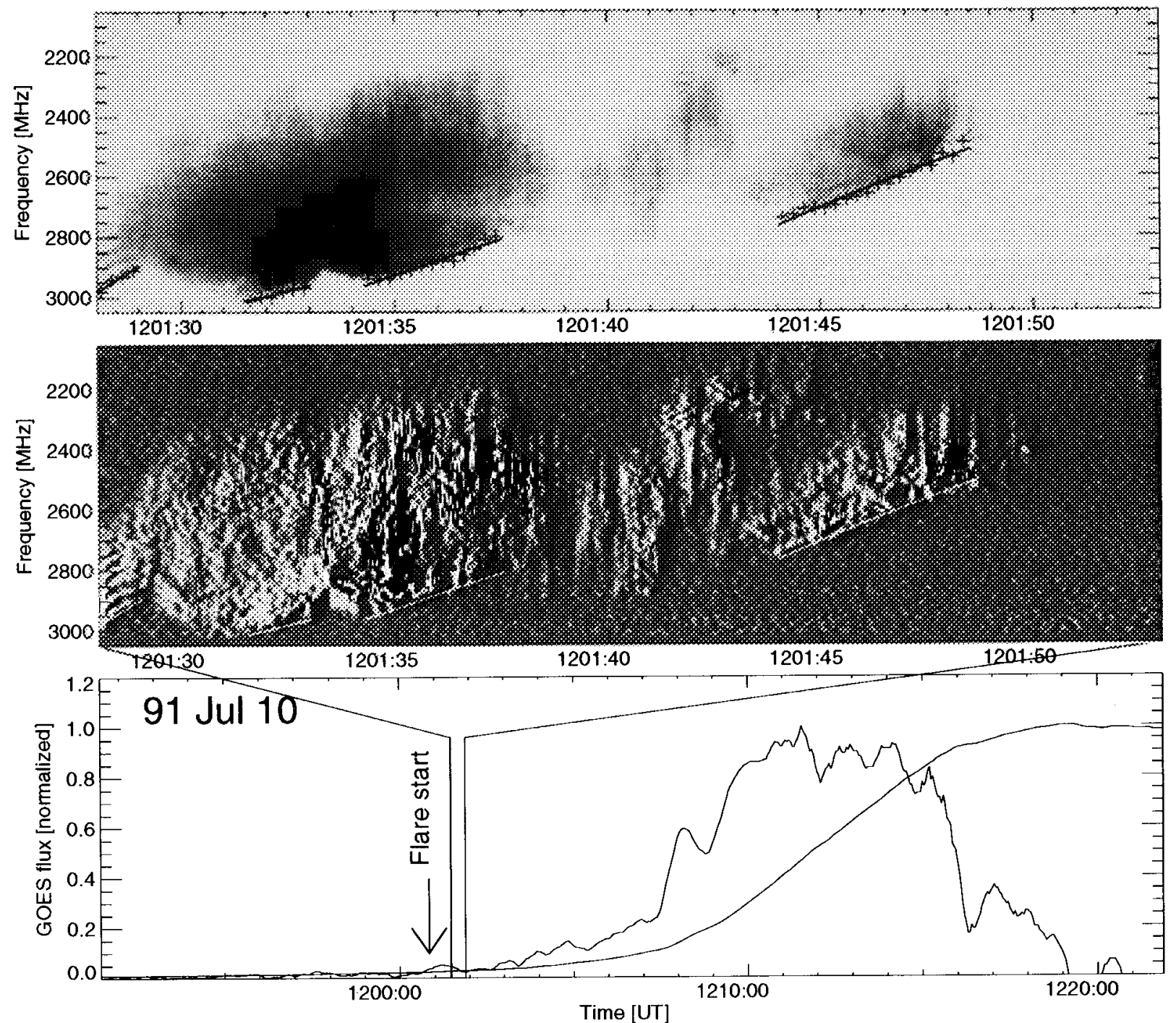

FIG. 7.- Radio flux $F_{R}(t o p)$ and time derivative $d F_{R} / d t$ (middle) of Phoenix data from the flare on 1991 July 10, $1201 \mathrm{UT}$, in the frequency range of $2-3 \mathrm{GHz}$. The gradient-filtered dynamic spectrum (middle) shows fine structure with fast drift bursts and slowly drifting parallel bands. In the four time segments (marked with bars), drift rates of $-90,-36,-50$, and $-61 \mathrm{MHz} \mathrm{s}^{-1}$ are measured at the high-frequency cutoff. The bottom frame shows the $0.5-4 \AA$ SXR flux and time derivative from GOES. The flare start is indicated at 1200:50 UT. Note that the radio data extend over a interval of $25 \mathrm{~s}$, while the GOES data are shown for a much larger interval of 35 minutes. 
of flares, with $n_{e}=0.6-1.0 \times 10^{11} \mathrm{~cm}^{-3}$ and $v_{\mathrm{CE}}=270 \mathrm{~km} \mathrm{~s}^{-1}$ (Table 1).

Our interpretation of this radio event in terms of chromospheric evaporation explains several observational properties: (1) the high-frequency cutoff is consistent with free-free absorption caused by the density enhancement (around $10^{11} \mathrm{~cm}^{-3}$ ) of a chromospheric evaporation front, (2) the slow negative drift rate is consistent with an upward motion of the evaporation front with a speed of $\$ 150 \mathrm{~km} \mathrm{~s}^{-1}$, and (3) the time delay of $\approx 36 \mathrm{~s}$ between the flare onset in SXR and the observed frequency cutoff at $3 \mathrm{GHz}$ is consistent with the travel time of the chromospheric evaporation front upward to an altitude $(\approx 6.0$ $\mathrm{Mm}$ ) corresponding to the plasma frequency level of $3 \mathrm{GHz}$ in our model (see Fig. 2). This interpretation offers also a number of additional new insights: (1) the emission mechanism is uniquely identified as (fundamental) plasma emission, (2) the spectral peak of the radio emission is explained by the reduced free-free opacity due to the high temperature $\left(\gtrsim 10^{7} \mathrm{~K}\right)$ at the front of the upflowing plasma, (3) the parallel drifting "zebra" bands represent a wave pattern comoving with the density or temperature structure of the chromospheric evaporation front, perhaps associated with supersonic shock propagation, (4) the parallel displacement of the high-frequency cutoffs between the subsequent five episodes indicates either sequential injections or spatial separation of multiple chromospheric evaporation cycles.

\subsubsection{Decimetric Pulsation Event}

Radio and HXR data of the flare of 1992 October 5 are shown in Figure 8. The displayed time interval of 0923:230926:18 UT corresponds to the recording time of Phoenix after a burst trigger and is almost coincident with the time interval when BATSE/CGRO detected $\geq 25 \mathrm{keV}$ HXRs, (i.e., 0923:250926:24 UT). SXRs detected by GOES start 43 s earlier than the HXRs, according to the first significant increase in the time derivative. An $\mathrm{H} \alpha$ subflare was registered in NOAA AR 7305 at heliographic position N12E41 (Solar Geophysical Data).
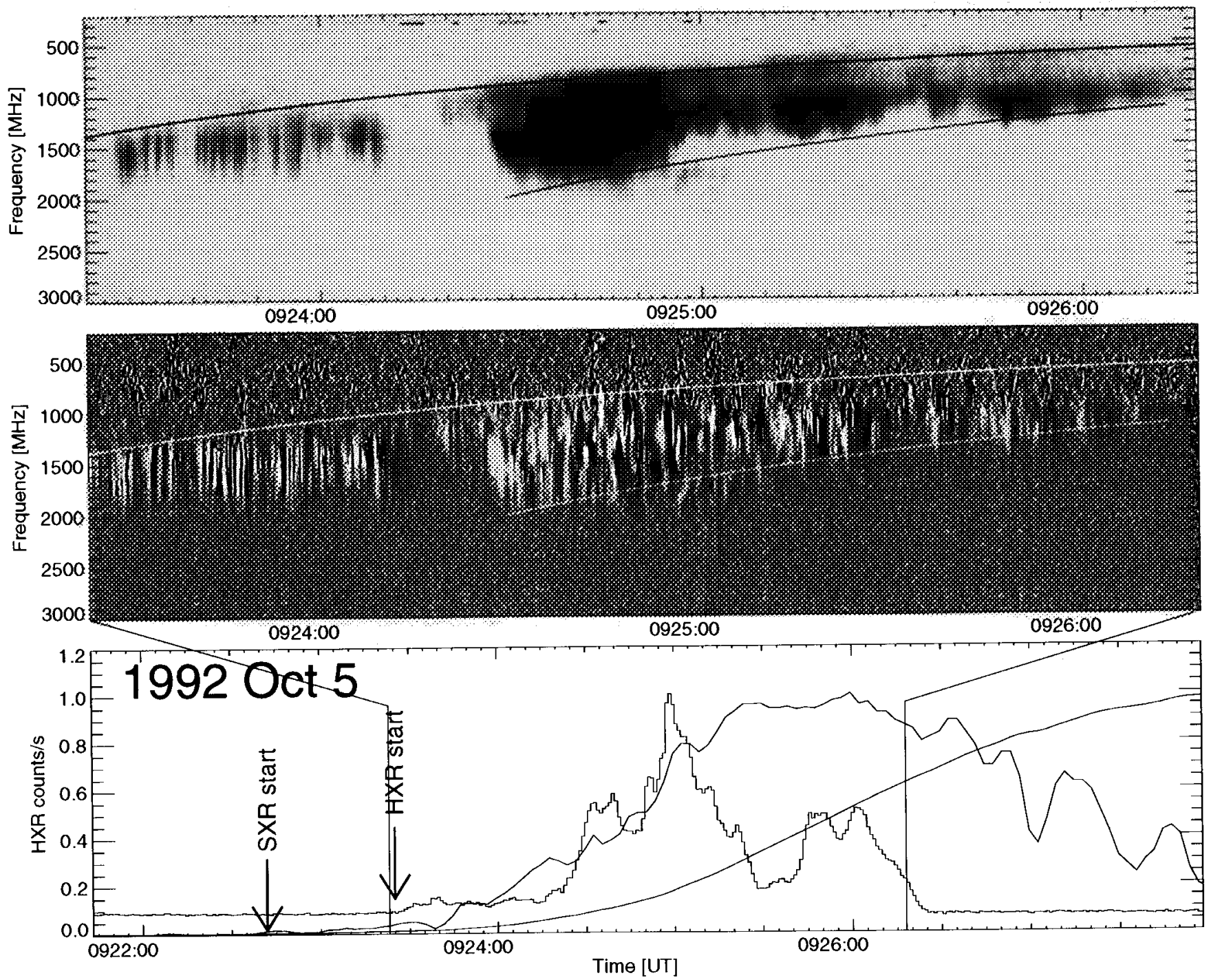

FIG. 8.-Flare of 1992 October 5, 0924 UT. The Phoenix radio data are shown background-subtracted (top), and gradient-filtered (middle). Both the low- and high-frequency cutoffs show a negative drift rate, -14 and $-17 \mathrm{MHz} \mathrm{s}^{-1}$ respectively, at the start of the fitted trajectory. The bottom panel shows $\mathrm{HXR}$ data $\geq 25$ $\mathrm{keV}$ from $B A T S E / C G R O$ (histogram), SXR data at $0.5-4 \AA$ from GOES (monotonically increasing curve), and the time derivative of the GOES data (fluctuating curve). 
The radio spectra show pulsating emission confined to the frequency range of $1.0-2.0 \mathrm{GHz}$. Below $1 \mathrm{GHz}$ the bandwidth of individual channels is $1 \mathrm{MHz}$ (vs. $10 \mathrm{MHz}$ above $1 \mathrm{GHz}$ ), to avoid interference. This enhances the receiver noise by a factor of $\sqrt{10}$ and reduces the sensitivity. Both the high- and lowfrequency cutoff show a slow negative drift rate, which was determined to be -14 and $-17 \mathrm{MHz} \mathrm{s}^{-1}$ at the start of the time segments indicated in Figure 8. This corresponds to velocities of 54 and $93 \mathrm{~km} \mathrm{~s}^{-1}$ according to our density model. The evolution of the dynamic spectrum has a remarkable similarity with the simulated cases in Figure 5: (1) in the first $50 \mathrm{~s}$ after the flare onset the high-frequency cutoff of the radio emission is almost constant; (2) after $50 \mathrm{~s}$ (during the strongest impulsive rise of the HXRs) the radio start frequency shifts to a higher value and (3) then drifts slowly toward lower frequencies. The exciter velocity associated with the slowly drifting start frequency $\left(93 \mathrm{~km} \mathrm{~s}^{-1}\right)$ is lower than expected for a typical chromospheric evaporation front $\left(300-400 \mathrm{~km} \mathrm{~s}^{-1}\right)$, probably because the drift of the start frequency is evaluated over a time interval (104 s) that is much longer than elementary peaks in HXR (with timescales of 5-10 s) and therefore represents an envelope encompassing multiple injected evaporation fronts, similar to the case of multiple injections observed in Ca XIX (Antonucci, Dodero, \& Martin 1990). It is also interesting to note that the low- and high-frequency cutoffs of the radio emission have an average ratio of $1: 2$, which corresponds to a density increase by a factor of 4 . This is about the density increase that is expected for a chromospheric evaporation front, inferred from the densities of a flare loop at the start and end of the flare (see Table 1).

\subsubsection{Decimetric Type III Events}

In Figure 9 we show four short episodes of type III burst groups that exhibit a systematic, drifting high-frequency cutoff. These short episodes with durations of $10-15 \mathrm{~s}$ are more likely to display single evaporation cycles, while the other events shown in Figures 7 and 8 contain multiple evaporation cycles. The high-frequency cutoff of those longer events is likely to encompass a number of shorter episodes, which cannot always be disentangled properly. The drift rate of the high-frequency envelope is then systematically lower, which leads to an underestimate of the velocity of the chromospheric evaporation front
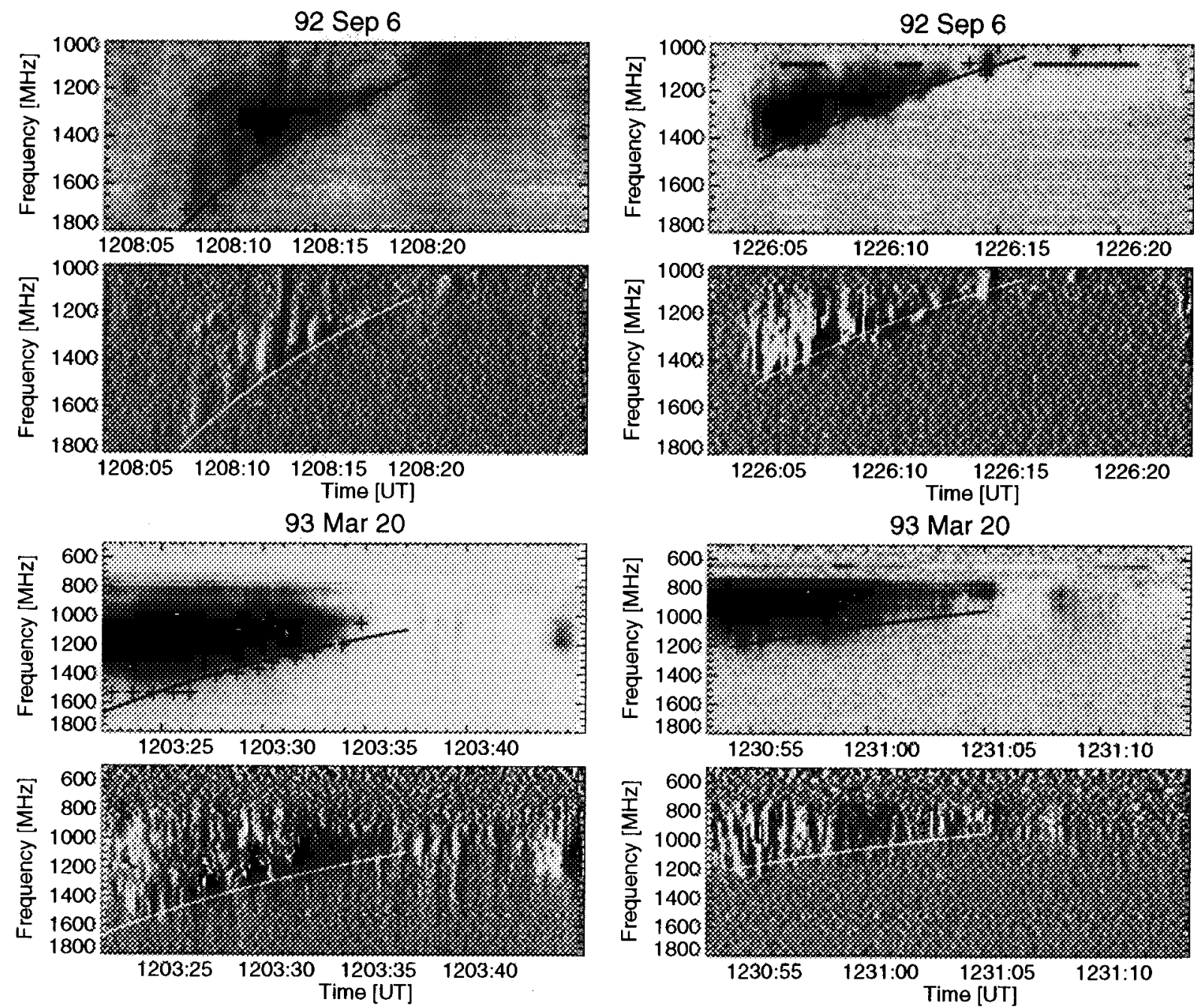

FIG. 9.-Four episodes with type III emission and a drifting high-frequency cutoff from the flares on 1992 September 6 (top rows) and 1993 March 20 (bottom

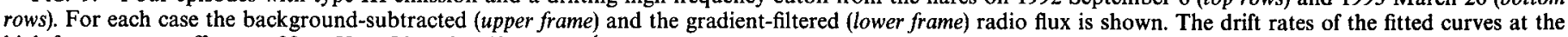
high-frequency cutoffs are $-82,-53,-56$, and $-42 \mathrm{MHz} \mathrm{s}^{-1}$. 
TABLE 3

Drift Rate and Velocity Measurements of High-Frequency Cutoffs

\begin{tabular}{|c|c|c|c|c|c|c|c|}
\hline Date & $\begin{array}{c}t_{0} \\
(\mathrm{UT})\end{array}$ & $\begin{array}{l}D \\
(\mathbf{s})\end{array}$ & $\begin{array}{c}v_{0} \\
(\mathrm{MHz})\end{array}$ & $\begin{array}{c}d v / d t\left(t_{0}\right) \\
\left(\mathrm{MHz} \mathrm{s}^{-1}\right)\end{array}$ & $\begin{array}{c}h_{0} \\
(\mathrm{Mm})\end{array}$ & $\left(\begin{array}{c}v_{0} \\
\left(\mathrm{~km} \mathrm{~s}^{-1}\right)\end{array}\right.$ & $\left(10^{10^{n_{e 0}}} \mathrm{~cm}^{-3}\right)$ \\
\hline 1991 Jul $10 \ldots \ldots$ & $1201: 28$ & 1.0 & 2982 & -90.1 & 6.0 & 153 & 11.0 \\
\hline $1991 \mathrm{Jul} 10 \ldots \ldots$ & $1201: 31$ & 1.5 & 3014 & -36.0 & 6.0 & 60 & 11.2 \\
\hline $1991 \mathrm{Jul} 10 \ldots \ldots$ & $1201: 34$ & 3.3 & 2962 & -49.9 & 6.1 & 85 & 10.9 \\
\hline 1991 Jul $10 \ldots \ldots$. & $1201: 44$ & 4.5 & 2765 & -61.3 & 6.4 & 120 & 9.5 \\
\hline 1992 Sep $05 \ldots \ldots$ & $1125: 33$ & 13.0 & 1878 & -15.3 & 8.9 & 60 & 4.4 \\
\hline 1992 Sep $06 \ldots \ldots$ & $0903: 26$ & 49.0 & 2079 & -14.0 & 8.2 & 46 & 5.4 \\
\hline 1992 Sep $06 \ldots \ldots$ & $1208: 08$ & 12.0 & 1786 & -82.4 & 9.3 & 360 & 4.0 \\
\hline 1992 Sep $06 \ldots \ldots$ & $1226: 05$ & 11.0 & 1484 & -53.0 & 10.9 & 325 & 2.7 \\
\hline 1992 Sep $06 \ldots \ldots$. & $1226: 44$ & 13.0 & 1157 & -5.4 & 13.4 & 53 & 1.7 \\
\hline 1992 Oct $05 \ldots .$. & 0924:29 & 104.0 & 2106 & -16.7 & 8.1 & 54 & 5.5 \\
\hline 1992 Oct $30 \ldots \ldots$ & $1132: 44$ & 40.0 & 1327 & -7.7 & 11.9 & 58 & 2.2 \\
\hline 1993 Mar $20 \ldots \ldots$ & $1203: 22$ & 15.0 & 1607 & -56.7 & 10.1 & 300 & 3.2 \\
\hline 1993 Mar $20 \ldots \ldots$ & $1205: 01$ & 23.0 & 2292 & -115.3 & 7.5 & 318 & 6.5 \\
\hline 1993 Mar $20 \ldots \ldots$ & $1209: 27$ & 45.0 & 2205 & -62.0 & 7.8 & 184 & 6.0 \\
\hline 1993 Mar $20 \ldots \ldots$ & $1217: 03$ & 42.5 & 1233 & -5.7 & 12.7 & 49 & 1.9 \\
\hline 1993 Mar $20 \ldots . .$. & $1230: 53$ & 10.0 & 1298 & -41.7 & 12.2 & 327 & 2.1 \\
\hline 1993 Mar 20...... & $1425: 30$ & 25.0 & 1704 & -27.4 & 9.7 & 130 & 3.6 \\
\hline 1993 Oct $02 \ldots \ldots$ & $0739: 50$ & 30.0 & 2147 & -23.1 & 8.0 & 72 & 5.7 \\
\hline 1993 Oct $02 \ldots \ldots$ & $0740: 28$ & 15.0 & 2104 & -70.6 & 8.1 & 228 & 5.4 \\
\hline 1993 Oct $02 \ldots \ldots$ & $0741: 26$ & 25.0 & 1586 & -8.2 & 10.3 & 44 & 3.1 \\
\hline 1993 Oct $07 \ldots \ldots$ & $1228: 52$ & 22.0 & 1424 & -9.6 & 11.2 & 64 & 2.5 \\
\hline Average ........... & $\begin{array}{l}\ldots \\
\ldots\end{array}$ & $\begin{array}{r}24.0 \\
\pm 23.3\end{array}$ & $\begin{array}{r}1960 \\
\pm 590\end{array}$ & $\begin{array}{l}-40.6 \\
\pm 31.7\end{array}$ & $\begin{array}{r}9.2 \\
\pm 2.3\end{array}$ & $\begin{array}{r}147 \\
+114\end{array}$ & $\begin{array}{r}5.2 \\
\pm 3.1\end{array}$ \\
\hline Average...$\ldots \ldots \ldots$ & $\begin{array}{c}(5<D<15 \mathrm{~s}) \\
\ldots\end{array}$ & $\begin{array}{r}12.7 \\
\pm 1.9\end{array}$ & $\begin{array}{r}1616 \\
\pm 330\end{array}$ & $\begin{array}{l}-46.4 \\
\pm 28.0\end{array}$ & $\begin{array}{r}10.4 \\
\pm 1.9\end{array}$ & $\begin{array}{r}236 \\
\pm 129\end{array}$ & $\begin{array}{r}3.4 \\
\pm 1.3\end{array}$ \\
\hline
\end{tabular}

(eq. [4]). Thus, the drift rates of the start frequency measured in shorter intervals such as those shown in Figure 9 are more likely to reveal the true velocity of resolved evaporation cycles. In the four cases in Figure 9 we measure drift rates of -82 , $-53,-57$, and $-42 \mathrm{MHz} \mathrm{s}^{-1}$. This corresponds to velocities of $360,325,300$, and $327 \mathrm{~km} \mathrm{~s}^{-1}$, which are fully consistent with the velocity range of $300-400 \mathrm{~km} \mathrm{~s}^{-1}$ deduced from Ca XIX lines.

\subsection{Statistics of Dynamical Parameters}

In Table 3 we summarize the measurements of all 21 analyzed events possessing a high-frequency cutoff, with the parameters measured at the beginning $\left(t_{0}\right)$ of the time intervals: the duration $(D)$, the frequency $\left(v_{0}\right)$, and the drift rate $(d v / d t)$. The density $\left(n_{e 0}\right)$ is derived for fundamental plasma emission. The velocity $\left(v_{0}\right)$ and altitude $\left(h_{0}\right)$ are inferred from the density model for overdense, type III-emitting flux tubes described above. The average velocity of all 21 measurements is $v_{0}=147$ $\pm 114 \mathrm{~km} \mathrm{~s}^{-1}$, and the average height is $h_{0}=9.2 \pm 2.3 \mathrm{Mm}$. However, the longer episodes probably contain a systematic error due to the cumulative effect of multiple evaporation cycles. On the other hand, events which are too short $(\lesssim 5 \mathrm{~s})$ have a larger uncertainty in the case of bursty emission. Thus, we consider the drift rates of start frequencies evaluated in intermediate time intervals, say in the range of 5-15 s, as most reliable. In Table 3 we find seven events with such durations, which imply a systematically higher value for the average upflow velocity; i.e., $v_{\mathrm{CE}}=236 \pm 129 \mathrm{~km} \mathrm{~s}^{-1}$.

\subsection{Statistics of Timing at Flare Onset}

We examine the timing of the first detection of a highfrequency cutoff in radio with respect to the flare start detected in either hard X-rays or soft X-rays. From the 21 events analyzed in Table 3 we find seven episodes that occur at a flare start. The start times of the radio events, $t_{0}$, are listed in Table 4. The start times of $\geq 25 \mathrm{keV} \mathrm{HXR}$ emission, $t_{\mathrm{HXR}}$, and the HXR peak count rate $\left(F_{\text {HXR }}\right)$ are taken from the BATSE/ CGRO catalog (compiled at SDAC/GSFC). The earliest start of SXR emission, $t_{\mathrm{SXR}}$, is evaluated from the first significant increase in the time derivative of the $3-25 \mathrm{keV}(0.5-4 \AA)$ GOES data. We exclude the event of 1993 October 7 from the statistics because the detected flare start seems to be severely

TABLE 4

Timing of X-Ray and Radio Signatures

\begin{tabular}{|c|c|c|c|c|c|c|c|}
\hline Date & $\begin{array}{c}t_{0} \\
\text { (UT) }\end{array}$ & $\begin{array}{l}t_{\text {SXR }} \\
\text { (UT) }\end{array}$ & $\begin{array}{c}t_{\mathrm{HXR}} \\
\text { (s) }\end{array}$ & $\begin{array}{c}F_{\text {HXR }} \\
{\text { (count } s^{-1}} \text { ) }\end{array}$ & $\begin{array}{c}t_{0}-t_{\mathrm{HXR}} \\
\text { (s) }\end{array}$ & $\underset{\text { (s) }}{t_{0}-t_{\text {SXR }}}$ & $\begin{array}{c}h_{0} / v_{0} \\
(\mathrm{~s})\end{array}$ \\
\hline 1991 Jul 10 & $1201: 28$ & $1200: 50$ & & & & 38 & 39 \\
\hline 1992 Sep 05 & $1125: 33$ & $1122: 30$ & $1122: 03$ & 95362 & 210 & 183 & 148 \\
\hline $1992 \operatorname{Sep} 06 \ldots \ldots$. & $0903: 26$ & $0900: 25$ & $0901: 45$ & 99873 & 101 & 181 & 178 \\
\hline 1992 Oct $05 \ldots \ldots$ & $0924: 29$ & $0922: 42$ & $0923: 25$ & 24079 & 64 & 107 & 150 \\
\hline 1993 Mar $20 \ldots \ldots$ & $1203: 22$ & $1202: 05$ & $1202: 08$ & 25968 & 74 & 77 & 34 \\
\hline 1993 Oct $02 \ldots \ldots$ & $0739: 50$ & $0739: 20$ & 0739:29 & 316913 & 21 & 30 & 111 \\
\hline 1993 Oct $07 \ldots \ldots$ & $1228: 52$ & $1228: 45$ & $1228: 55$ & 1686 & -3 & 7 & 175 \\
\hline \multirow[t]{2}{*}{ Average $\ldots \ldots \ldots \ldots$} & $\cdots$ & $\ldots$ & $\ldots$ & $\ldots$ & 94 & 103 & 110 \\
\hline & $\ldots$ & $\ldots$ & $\ldots$ & $\ldots$ & \pm 71 & \pm 67 & \pm 61 \\
\hline
\end{tabular}


affected by instrumental sensitivity limitations, since it has the lowest HXR count rate, being one to two orders of magnitude weaker than the other events.

From the other six events we find for the earliest detection of a radio high-frequency cutoff an average delay of $t_{0}-t_{\mathrm{HXR}}=$ $94 \pm 71 \mathrm{~s}$ with respect to the HXR onset, and $t_{0}-t_{\text {SXR }}=103$ $\pm \overline{67} \mathrm{~s}$ with respect to the SXR onset. We compare these delays with the expected propagation delay $\Delta t_{\mathrm{CE}}$ of the chromospheric evaporation front on its path up to the coronal height corresponding to the location of the radio source, using the parameters $h_{0}$ and $v_{0}$ from Table 3 (i.e., $\Delta t_{\mathrm{CE}}=h_{0} / v_{0}$ ). We find an average expected delay of $\Delta t_{\mathrm{CE}}=110 \pm 61 \mathrm{~s}$, which agrees well with the delay $t_{0}-t_{\text {SXR }}$ measured after the onset of SXRs $(103 \pm 67 \mathrm{~s})$.

\section{DISCUSSION}

We discuss three reasons that substantiate evidence for the radio detection of chromospheric evaporation at decimetric wavelengths: (1) the propagation velocity $(\S 4.1)$, (2) the electron density ( $\$ 4.2$ ), and (3) the timing with respect to HXRs and SXRs ( $\$ 4.3$ ). Accepting the consequences of the chromospheric evaporation scenario as modeled here, several models of decimetric radio bursts require a revised interpretation that includes the dynamics of high-temperature flare plasmas $(\S 4.4)$.

\subsection{Velocity Measurements}

The idea that the drift of the start frequency of decimetric radio bursts could be attributed to the chromospheric evaporation process was first considered in Aschwanden et al. (1993), where a velocity of $\approx 300 \mathrm{~km} \mathrm{~s}^{-1}$ was inferred for the exciter associated with the high-frequency cutoff of a type IV event with quasi-periodic fine structure.

In this study we investigated a variety of decimetric radio bursts (type IV, type IIIdm, broadband pulsations), which all exhibit an upward drifting start frequency. We also found a few rare cases with downward drifting high-frequency cutoffs during short intervals ( $\lesssim 5 \mathrm{~s}$ ) in some flares. Because HXR usually show major fluctuations on timescales of $\gtrsim 5 \mathrm{~s}$ (DeJager \& DeJonge 1978), indicative of multiple energy releases in potentially different locations, it is likely that multiple chromospheric evaporation cycles occur. Such evaporation cycles have been observed on timescales of $\approx 1$ minute by Antonucci et al. (1990). For time intervals $\gtrsim 1$ minute, it is likely that the averaged drift rate of the start frequency encompasses multiple injections and thus yields only a lower limit to the speed of an individual evaporation front. We consider the measurement of the start frequency over time intervals of 5-15 $s$ more suitable for measuring the true propagation speeds of individual chromospheric evaporation cycles. Based on the statistics presented in Table 3 , we find an average velocity of $v_{\mathrm{CE}}=236 \pm 129 \mathrm{~km}$ for the shorter time intervals. Also, we have to be aware that the calibration of the velocity measurement used here is of statistical nature, relying on our model based on statistical values of type III drift rates.

The drift rates of the detected high-frequency cutoffs are shown as a function of the frequency in Figure 10 for the 21 analyzed events. The cutoffs fall into a band between 50 and $350 \mathrm{~km} \mathrm{~s}^{-1}$. We assumed a constant velocity in our fitting procedure, which is justified considering the small altitude range over which a fit extends. However, our velocity is measured at a height of $\approx 10 \mathrm{Mm}$, while soft X-ray blueshifts in $\mathrm{Ca}$ XIX are weighted by the emission measure along the line of

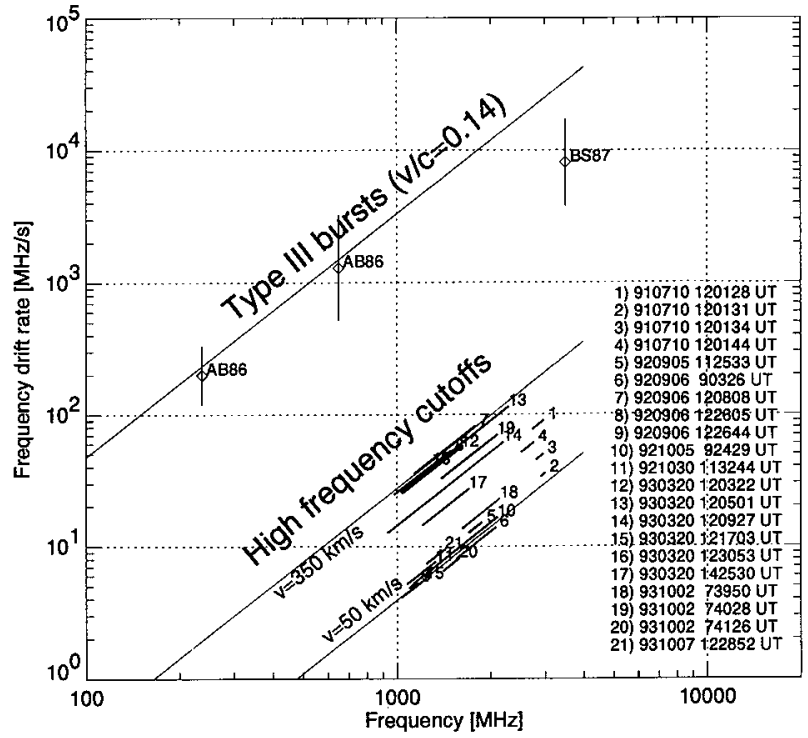

Fig. 10.-Drift rates of type III bursts and high-frequency cutoffs. The average drift rate (diamonds) and standard deviation (error bar) of type III bursts are taken from Aschwanden \& Benz (1986) (AB86) and Stähli \& Benz (1987) (BS87). The upper line, labeled with $v / c=0.14$, corresponds to the average drift rate of type III bursts, specified by the relation $d v / d t=-0.01$ $v^{1.84}$ of Alvarez \& Haddock (1973). The lower straight lines, marking a velocity range of $50-350 \mathrm{~km} \mathrm{~s}^{-1}$, encompass the drift rates measured at the highfrequency cutoffs of 21 flare episodes, labeled with date and start time on the right.

sight. Since the centroid of the Ca XIX emission is probably formed below $10 \mathrm{Mm}$, certainly during the first $30 \mathrm{~s}$ of the flare, the SXR-derived velocities apply to lower heights than in radio.

\subsection{Electron Density Measurements}

Radio emission at the plasma frequency provides the most direct measurement of the local electron density of a plasma. If radio type III bursts are observed, the only ambiguity in the local density measurement of the type III source is whether the radio waves are emitted at the fundamental $(s=1)$ or harmonic $(s=2)$ plasma frequency. This ambiguity in the frequency $\left(v=s v_{p} \propto s \sqrt{n_{e}}\right)$ causes a factor of four uncertainty in the electron density. If we are to use the high-frequency cutoff of plasma emission as a density diagnostic of the chromospheric evaporation front, we have to resolve this ambiguity. Fortunately, there are three strong arguments that the observed high-frequency cutoff applies to fundamental plasma emission: (1) our model of overdense, type III-emitting flux tubes, based on the statistically observed type III drift rate, was found to be consistent with an average exciter speed of $v_{\mathrm{III}} / c=0.14$ in the case of fundamental plasma emission, which is consistent with the exciter speed $v_{\mathrm{III}} / c=0.15 \pm 0.04$ derived from absolute velocity measurements, while harmonic emission yields a less satisfactory agreement; (2) the mean velocity of chromospheric evaporation fronts inferred from our density model agrees better with the measurements of the Ca XIX line in the case of fundamental emission (within a factor of 1.1) than for the case of harmonic emission (within a factor of 1.8); (3) the free-free opacity is able to produce a steep high-frequency cutoff for fundamental plasma emission in the $1-3 \mathrm{GHz}$ frequency range, while no reasonable parameters can be found for harmonic plasma emission to produce the same effect (see simulated spectra in parameter space in Fig. 6). Thus, we conclude that 
the slowly drifting high-frequency cutoff observed here is likely to be produced at the fundamental plasma frequency level. This gives us a very accurate tool to probe the electron density at the chromospheric evaporation front. For microwave type III bursts observed at $3.5 \mathrm{GHz}$ (Stähli \& Benz 1987) and at 6.2-8.4 GHz (Benz et al. 1992), harmonic plasma emission was proposed based on the scale height argument derived from the observed drift rate, leading to a relatively low coronal temperature (0.5-0.7 MK) if the flux tube is assumed to be in isothermal equilibrium. In the present study we are forced to abandon the assumption of isothermal equilibrium in flaring flux tubes, especially in the environment of chromospheric evaporation. The high temperature $(\approx 17 \mathrm{MK})$ of the hot upflowing plasma reduces the free-free opacity drastically (because of the $T^{-3 / 2}$ dependence) and makes overdense flux tubes transparent to fundamental plasma emission, even at microwave frequencies.

Our measurements indicate an electron density of $n_{e}=$ $5.2( \pm 3.1) \times 10^{10} \mathrm{~cm}^{-3}$ at the time of first detection of the chromospheric evaporation in radio, at an average height of $h=9.2 \pm 2.3 \mathrm{Mm}$. From the emission measure of $\mathrm{Ca} \mathrm{XIX}$, similar electron densities of $5.6-9.6 \times 10^{10} \mathrm{~cm}^{-3}$ (Table 1) are derived at the onset of the flare and typically increase by a factor of 4 during the flare. We have to take into account that the densities derived from the $\mathrm{Ca}$ XIX line represent averages over the column depth below the chromospheric front, while the radio method measures the density directly at the top of the chromospheric evaporation front and thus returns a lower value.

\subsection{Timing of $X$-Ray and Radio Signatures}

The earliest detection of chromospheric evaporation indicated by a blueshifted Ca XIX line generally coincides with the start of $\geq 25 \mathrm{keV}$ HXRs (see Fig. 5 in Antonucci et al. 1993). If we take the start of $\geq 25 \mathrm{keV}$ HXRs as a proxy for the first start of chromospheric evaporation, we can test the timing of the earliest detection in radio by the start time of the highfrequency cutoff. According to the statistics presented in Table 4 , we find the first radio detection of a decimetric highfrequency cutoff $94 \pm 71 \mathrm{~s}$ after the start of enhanced $\geq 25 \mathrm{keV}$ HXR emission or $103 \pm 67 \mathrm{~s}$ after the first significant increase in the rate of 3-15 keV SXR emission. The time difference between HXR emission and the time derivative of SXR emission should be small for those flares where the Neupert effect (Dennis \& Zarro 1993) applies and is indeed negligible here $(9 \mathrm{~s})$ compared with the delay times ( 94 or $103 \mathrm{~s}$ ) of the radio onset. The onset of HXR or SXR corresponds to the launch of a chromospheric evaporation front down in the chromosphere, while the radio detection takes place at some height in the corona, and thus, a natural propagation delay is expected. Within the framework of our density model, we determined the altitudes $h_{0}$ and the velocities $v_{0}$ of the upflowing plasma selfconsistently from the frequency and drift rate of the highfrequency cutoff. An interesting test is now to compare the extrapolated propagation times $h_{0} / v_{0}$ with the actual delays. From the statistics presented in Table 4, we find an average extrapolated propagation delay of $h_{0} / v_{0}=110 \pm 61 \mathrm{~s}$, which is in good agreement with the actual detection delay of $t_{0}$ $-t_{\mathrm{SXR}}=103 \pm 67$ in radio with respect to the start in SXR. Thus, this temporal coincidence strongly supports a causal connection between the launch of a chromospheric evaporation front detected in SXR and the detection of a highfrequency cutoff in radio.
Antonucci et al. (1990) identified successive injections of evaporating plasma on timescales of minutes, where each evaporation cycle showed initially a high velocity and decreased progressively during a cycle. Similarly, we identified multiple high-frequency cutoffs during a flare. The 21 episodes listed in Table 3 show a case with up to five episodes, which indicates multiple evaporation cycles. The intervals between successive episodes were found to be as short as $3 \mathrm{~s}$ (e.g., in 1991 July 10 flare; Fig. 7), but it is not clear whether the multiple frequency cutoffs refer to temporally successive evaporation cycles or to different propagation delays resulting from different paths (e.g., adjacent flux tubes).

\subsection{Implications for Metric and Decimetric Radio Bursts}

Our chromospheric evaporation scenario predicts for times $\gtrsim 20$ s after flare start (1) a slowly-drifting high-frequency (and possibly also a low-frequency) cutoff and (2) a significantly higher drift rate (than expected for the isothermal quiet corona) for decimetric bursts emitted at the fundamental plasma frequency. At first glance, a number of decimetric bursts can be found which match these morphological criteria: e.g., decimetric type III bursts (Figs. 3, 5, and 10 in Isliker \& Benz 1994), type IV bursts (Figs. 24, 25, and 26 in Isliker \& Benz 1994), and broadband pulsations (Figs. 28, 29, and 30 in Isliker \& Benz 1994). Our chromospheric evaporation model sheds some new light on the interpretation of such burst types. As an example, we discuss the case of type III bursts with "infinite drift rates."

The passage of a chromospheric evaporation front at frequencies of $200-500 \mathrm{MHz}$ may have also been observed by Poquerusse (1994). In the examples of fast-drifting type III bursts shown in Figures 1 and 3 in Poquerusse (1994) there appears at $\approx 200 \mathrm{MHz}$ a slowly upward drifting separatrix, which divides the normal-drifting metric type III bursts from the significantly faster drifting decimetric extensions. In our scenario this is naturally explained by the steeper density gradient at the chromospheric evaporation front which drifts slowly toward lower frequencies. Poquerusse (1994) proposes an alternative interpretation in terms of highly relativistic $\left(v_{\mathrm{III}} / c \gtrsim 0.8\right)$ electron beams and suggests that these fast-drift bursts constitute a class separate from normal type III bursts. Since the chromospheric evaporation model provides a natural explanation for the transition of normal-drifting to fast-drifting type III bursts at decimetric frequencies, we do not see a strong reason to involve relativistic exciters. Poquerusse's two classes of type III bursts may simply be renamed in type III bursts above and below the interface of a chromospheric evaporation front.

\section{SUMMARY AND CONCLUSIONS}

\subsection{Observational Summary}

We analyzed the radio dynamic spectra in the $0.1-3.0 \mathrm{GHz}$ range from Phoenix, simultaneous HXR data from BATSE/ $C G R O$, and SXR data from GOES, from the years 1991-1993. We identified 21 time intervals during 11 different flares wherein the radio emission shows a high-frequency cutoff in the decimetric range, either in the form of a sharp spectral drop-off of continuum emission, or in form of a systematic change in the start frequency of bursty emission. The observational results (see details in Tables 3 and 4) are as follows:

1. The high-frequency cutoff during these 21 events shows a slow negative drift rate, with an average of $(d v / d t)_{0}=-41$ 
$\pm 32 \mathrm{MHz} \mathrm{s}^{-1}$, measured at an average start frequency of $v_{0}=1960 \pm 590 \mathrm{MHz}$

2. The electron density of the average start frequency where the radio high-frequency cutoffs is first detected is $n_{e 0}=$ $5.2( \pm 3.1) \times 10^{10} \mathrm{~cm}^{-3}$ (assuming fundamental plasma emission). This value is close to the value of $n_{e}=5.6-9.6$ $\times 10^{10} \mathrm{~cm}^{-3}$ inferred from the (blueshifted) $\mathrm{Ca}$ XIX emission measure.

3. The start time $t_{0}$ of the first detected high-frequency cutoff in a flare was found to be delayed by $t_{0}-t_{\mathrm{HXR}}=94$ $\pm 71 \mathrm{~s}$ with respect to the start of $\geq 25 \mathrm{keV} \mathrm{HXR}$ emission, and by $t_{0}-t_{\mathrm{SXR}}=103 \pm 67 \mathrm{~s}$ with respect to the start of the 3-25 keV SXR emission.

\subsection{Summary of Model-dependent Data Analysis}

We established a coronal density model for overdense, type III-emitting flare loops, consistent with the statistically observed drift rate of type bursts in the lower corona (powerlaw relation of Alvarez \& Haddock 1973) and with an isothermal, barometric model in the upper corona. This model was found to be consistent with a statistical exciter speed of $v_{\mathrm{III}} / c=$ 0.14 for type III bursts radiating at the fundamental plasma frequency, in agreement with the average value obtained from imaging observations, $v_{\mathrm{III}} / c=0.15 \pm 0.04$. Based on this model we find the following results:

4. The exciter velocity associated with the slowly drifting high-frequency cutoffs corresponds to $v_{0}=147 \pm 114 \mathrm{~km} \mathrm{~s}^{-1}$ for all episodes, or $v_{0}=236 \pm 129 \mathrm{~km} \mathrm{~s}^{-1}$ for episodes with 5-15 s duration. In comparison, the velocity of the chromospheric evaporation front inferred from the blueshifted $\mathrm{Ca}$ XIX line is $270 \pm 90 \mathrm{~km} \mathrm{~s}^{-1}$.

5. The altitude where the high-frequency cutoffs are first detected corresponds to $h_{0}=9.2 \pm 2.3 \mathrm{Mm}$.

6. The extrapolated start time of an exciter starting in the chromosphere and propagating with velocity $v_{0}$ up to a coronal height $h_{0}$ is found to be $h_{0} / v_{0}=110 \pm 61 \mathrm{~s}$ for the earliest episode in a flare. This value is close to the observed delay of the earliest detection of a radio high-frequency cutoff with respect to the onset of SXR, i.e., $t_{0}-t_{\mathrm{SXR}}=103 \pm 67 \mathrm{~s}$.

We interpret the slowly drifting high-frequency cutoff at decimetric wavelengths discovered here in terms of an opacity effect resulting from the upflowing hot flare plasma during the chromospheric evaporation process at the begin of the impulsive flare phase. Evidence for this interpretation is given by the good statistical agreement between the parameters inferred from radio and $\mathrm{Ca} \mathrm{XIX}$.

\subsection{Implications from Model Calculations}

We performed numerical calculations of the frequencydependent free-free absorption for a propagating source radiating at the fundamental or harmonic plasma frequency. The configuration of the simulated model is an overdense flux tube embedded in an upflowing hot plasma, where the exciter of plasma emission is assumed to propagate inside the cylindrical flux tube. The flux tube has a diameter of $2-10 \mathrm{Mm}$ and a coronal temperature $T_{C}=1.5 \mathrm{MK}$, and the chromospheric evaporation front is simulated by a four-fold density increase and a temperature of $T_{\mathrm{CE}} \approx 15 \mathrm{MK}$. The numerical results imply the following:

1. The density gradient at the chromospheric evaporation front causes a local discontinuity in the plasma frequency that produces an almost infinite drift rate for type III-like bursts over the corresponding part of the radio spectrum (estimated to be within a factor of $\approx 2$ in frequency).

2. The radio brightness is enhanced by a large factor of approximately $\left(T_{\mathrm{CE}} / T_{C}\right)^{(3 / 2)} \approx 32$ near the chromospheric evaporation front owing to the temperature dependence of the free-free opacity.

3. Above the frequency $v_{\mathrm{CE}}$, the free-free radio spectrum rapidly falls off for fundamental plasma emission due to the density dependence of the free-free opacity. Harmonic plasma emission is not much affected in the same parameter range. Thus, the observation of a steep high-frequency cutoff implies fundamental plasma emission.

4. The high-frequency cutoff for plasma emission is steeper for (1) lower chromospheric evaporation temperatures, (2) limb flares, or (3) small flux tube diameters.

5 . The high-frequency cutoff drifts toward lower frequencies, at a rate proportional to the velocity of the chromospheric front.

Isothermal, hydrostatic equilibrium is an invalid assumption in the environment of chromospheric evaporation and should be abandoned when deriving the local electron density scale height from the drift rate of decimetric bursts, in particular for the fast-drifting decimetric bursts commonly detected during the impulsive phase of flares.

\subsection{Consequences for Future Work}

We have established a statistical correspondence between the SXR and decimetric signatures of chromospheric evaporation. The interpretation of decimetric radio emission during the impulsive flare phase should be reconsidered under this new aspect. The dynamics of the high-temperature plasma have been found to have a profound influence on the free-free opacity of decimetric plasma emission. In particular, we have demonstrated a new method for including the evolution of the upflowing hot flare plasma in physical models that can fit the temporal and spectral evolution of decimetric radio bursts. While this study is focused on statistical parameters to establish the first evidence for a relationship between the phenomena observed in SXR and radio, we anticipate that future studies with simultaneous measurements in both wavelength regimes will allow a detailed modeling of individual flares. The BCS spectra from Yohkoh and simultaneous broadband dynamic spectra from Phoenix complement each other in an ideal way for this purpose.

Studying chromospheric evaporation at radio wavelengths offers several advantages: (1) chromospheric evaporation can be detected from ground-based experiments, in contrast to soft $\mathrm{X}$-ray measurements which require a spaceborne detector; (2) the plasma frequency measured in radio offers a very accurate density diagnostic of the upflowing plasma and complements the density measurement in SXR lines based on the line-ofsight integrated emission measure of the density-sensitive emission lines; (3) the spectral shape at high-frequency cutoffs offers a sensitive temperature diagnostic: free-free absorption is especially sensitive to admixtures of cooler plasma ( $T \lesssim 1 \mathrm{MK}$ ), while current SXR detectors are generally not sensitive in this temperature regime; (4) no center-to-limb effect is expected for the velocity measurements inferred from drift rates at radio wavelengths, thus allowing velocity measurements even near the limb. This is not possible with the Doppler-shift method in $\mathrm{Ca}$ XIX. On the other hand, blueshift measurements with the 
Ca XIX line offer a more accurate velocity measurement that can be used as a calibration of the density model.

We found that the consequences of chromospheric evaporation are most effective for the decimetric range ( $\gtrsim 300 \mathrm{MHz}$ ). The expected effects at metric wavelengths are retarded by several minutes, the exciter velocity is slowed down, and the radiative signatures are perhaps diluted due to spatial dispersion and merging of subsequent cycles. Possible related metric phenomena are type II bursts (shock waves), moving type IV bursts, or type I continua depressions (Böhme \& Krüger 1982; Aurass et al. 1993).

Finally, we would also like to point out that the radio detection of upflowing, hot chromospheric plasma might be possible for stellar flares. Since coherent radio emission requires many fewer energetic particles than does incoherent (thermal) emission at SXR wavelengths, the slowly drifting high-frequency cutoff of plasma emission should be easier to detect than a blueshift in SXR lines. Thus, the method described offers the possibility of detecting hot plasma upflows in stellar flares and to probe density models of stellar coronae.

Helpful discussions with Keith Strong, Dominic Zarro, and Julia Saba and careful reviewing by Jeff de La Beaujardière are gratefully acknowledged. The authors are thankful for access and support of BATSE/CGRO and GOES data archives at the Solar Data Analysis Center at NASA/GSFC. The work of M. J. A. was supported at GSFC and UMd by NASA grants NAS 5-30442, NAGW-3080, NAGW-3456, NAG 5-2001, and NAG 5-2352. A. O. B. and the construction of the radio spectrometer Phoenix was supported by grant 20-34045.92 from the Swiss National Science Foundation.

\section{APPENDIX A}

\section{DENSITY MODEL FOR TYPE III-EMITTING FLARE LOOPS}

We construct a density model for flare loops which is (1) consistent with the statistically measured drift for type III bursts (eq. [2]; Alvarez \& Haddock 1973) in the lower corona and (2) has a continuous transition to the isothermal, barometric atmosphere in the upper corona. This can be achieved by assuming a power-law function in the lower corona and an exponential form in the upper corona, i.e.,

$$
n_{e}(h)= \begin{cases}n_{1}\left(\frac{h}{h_{1}}\right)^{-p} & h<h_{1}, \\ n_{Q} \exp \left(-\frac{h}{\lambda}\right) & h>h_{1},\end{cases}
$$

with a transition at height $h_{1}$. The barometric model is constrained by the electron density $n_{Q}$ at the base of the quiet corona and the density scale height $\lambda$. A smooth transition between the two regimes is obtained by requiring that the function and its first derivative be continuous at the transition height $h=h_{1}$. These continuity conditions determine the transition height $h_{1}$ and density $n_{1}=$ $n_{e}\left(h=h_{1}\right)$ at this height, i.e.,

$$
h_{1}=p \lambda, \quad n_{1}=n_{Q} \exp (-p) \text {. }
$$

For the lower corona $\left(h<h_{1}\right)$ we calculate the dynamic spectrum $v(t)$ for the case of plasma emission. The observed frequency $v$ is assumed to be emitted at the sth harmonics of the plasma frequency $v_{p}$, and we can relate the frequencies to the electron densities according to (in cgs units)

$$
v=s v_{p}, \quad v_{p}=\left(\frac{n_{e} q_{e}^{2}}{\pi m_{e}}\right)^{1 / 2}=8980 n_{e}^{1 / 2}, \quad v_{Q}=\left(\frac{n_{Q} q_{e}^{2}}{\pi m_{e}}\right)^{1 / 2}=8980 n_{Q}^{1 / 2}
$$

For an upward-moving exciter with constant velocity $v$, starting at time $t_{0}$ and height $h_{0}$, the altitude $h(t)$ can be parameterized as a function of time $t$ by

$$
h(t)=h_{0}+v\left(t-t_{0}\right)
$$

Thus, for $h<h_{1}$,

$$
v(t)=s v_{Q}\left[\frac{e}{p} \frac{h_{0}+v\left(t-t_{0}\right)}{\lambda}\right]^{-p / 2}
$$

The time derivative of this dynamic spectrum yields the drift rate

$$
\frac{\partial v}{\partial t}=-\frac{e v\left(s v_{Q}\right)^{-2 / p}}{2 \lambda} v^{(1+2 / p)} .
$$

Comparison with the empirical drift rate law of Alvarez \& Haddock (1973), observed in the frequency range of 75-550 $\mathrm{MHz}$, i.e., $\partial v / \partial t=-A v^{\alpha}$, with $\alpha=1.84$, and $A_{\mathrm{MHz}}=0.01$ if the frequency is measured in $\mathrm{MHz}$, or $A=9 \times 10^{-8}$ if measured in $\mathrm{Hz}$, we find the following relations for the constants $A$ and $\alpha$ :

$$
\alpha=\left(1+\frac{2}{p}\right), \quad A=\frac{e v\left(s v_{Q}\right)^{-2 / p}}{2 \lambda}
$$


This determines the remaining constants $p$ and $n_{Q}$ in equation (A1):

$$
p=\frac{2}{\alpha-1}, \quad n_{Q}=\left(\frac{e v}{2 A \lambda}\right)^{p}(s 8980)^{-2} .
$$

Using the Baumbach-Allen model for the quiet corona, we find $n_{Q}=n_{e}^{\mathrm{BA}}(h=0)=4.6 \times 10^{8} \mathrm{~cm}^{-3}$. The scale height of the barometric model is determined by the temperature of the quiet corona $\left(T_{Q}=1.5 \mathrm{MK}\right)$, given by the relation $\lambda=4.6 \times 10^{9} T_{Q}(\mathrm{MK})$. The numerical values for the constants in the density model $n_{e}(h)$ (eq. [A1]) are then

$$
p=2.38, \lambda=6.9 \times 10^{9} \mathrm{~cm}, \quad h_{1}=1.6 \times 10^{10} \mathrm{~cm}, \quad n_{Q}=4.6 \times 10^{8} \mathrm{~cm}^{-3} .
$$

Since this density model entirely relies on the statistical relation found by Alvarez \& Haddock (1973), we check the consistency with recent measurements at higher frequencies. A comparison is shown in Figure 10. The centroid and width of drift rate distributions from 461 type III drift rates at $237 \mathrm{MHz}$, and from 294 type IIIdm drift rates at $650 \mathrm{MHz}$ (Aschwanden \& Benz 1986), are found to be very close to the relation of Alvarez \& Haddock (1973) (see symbols AB86 in Fig. 10). The average drift rate of 35 microwave type III bursts at $3.5 \mathrm{GHz}$ was found to be $8100 \pm 4300 \mathrm{MHz} \mathrm{s}^{-1}$ in Stähli \& Benz (1987). This value (indicated with symbols BS87 in Fig. 10) is a factor of 4 below the expected value from the Alvarez \& Haddock (1973) relation.

\section{APPENDIX B}

\section{THE EXCITER SPEED OF TYPE III BURSTS}

The mean type III exciter velocity that is most consistent with our model inferred from the drift rate relation of Alvarez \& Haddock (1973) can be obtained from equation (A8):

$$
v_{\mathrm{III}}=\frac{2 A \lambda}{e}\left[n_{Q}(s 8980)^{2}\right]^{(1 / p)} .
$$

For the constants used above (eq. [A9]) we find $v_{\mathrm{III}} / c=0.14$ for fundamental $(s=1)$ plasma emission, or $v_{\mathrm{III}} / c=0.25$ for harmonic $(s=2)$ plasma emission. In the following, we test which value is more consistent with measured exciter speeds of type III bursts.

The values of measured exciter speeds of type III bursts compiled in Table 2 have been inferred with different methods that all have different systematic errors. Generally, there is a trend: a higher value was found in earlier studies using interferometric position measurements of type III sources, compared with interplanetary position measurements by later spacecrafts. Dulk et al. (1987) attribute this trend to the fact that (1) the apparent source location was not corrected for wave ducting, and (2) the density along the type III paths was overestimated in earlier measurements. It is beyond the scope of this work to evaluate the individual systematic errors of each measurement. Excluding the early measurements before 1970 which are believed to be systematically too high, we find an average value of $v_{\mathrm{II}} / c=0.15 \pm 0.04$, by weighting the values according to the number of independently analyzed bursts. This mean value is remarkably close to the value found in our model for fundamental plasma emission, $v_{\mathrm{III}} / c=0.14$. This constitutes also a new argument that most of these type III bursts are emitted at the fundamental plasma frequency.

Alvarez, H., \& Haddock, F. T. 1973, Solar Phys., 29, 197

Antiochos, S. K., \& Sturrock, P. A. 1978, ApJ, 220, 1137

Antonucci, E. 1989, Solar Phys., 121, 31

Antonucci, E., Dodero, M. A., \& Martin, R. 1990, ApJS, 73, 137

Antonucci, E., Dodero, M. A., Martin, R., Peres, G., Reale, F., \& Serio, S. 1993 , ApJ, 413, 786

Antonucci, E., Gabriel, A. H., Acton, L. W., Culhane, J. L., Doyle, J. G.

Leibacher, J. W., Machado, M. E., Orwig, L. E., \& Rapley, C. G. 1982, Sol. Phys., 78, 107

Antonucci, E., Gabriel, A. H., \& Dennis, B. R. 1984, ApJ, 287, 917

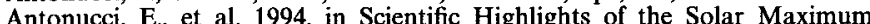
Mission: The Many Faces of the Sun, ed. K. T. Strong, J. L. R. Saba, \& B. M. Haisch (Berlin: Springer-Verlag), in press

Aschwanden, M. J., Bastian, T. S., Benz, A. O., \& Brosius, J. W. 1992, ApJ, 391 380

Aschwanden, M. J., \& Benz, A. O. 1986, A\&A, 158, 102

Aschwanden, M. J., Benz, A. O., Dennis, B. R., \& Gaizauskas, V. 1993, ApJ, 416,857

Aschwanden, M. J., Benz, A. O., \& Montello, M. L. 1994, ApJ, 431, 432

Aurass, H., Hofmann, A., Magun, A., Soru-Excaut, I., \& Zlobec, P. 1993, Solar Phys., 145, 151

Benz, A. O., Güdel, M., Isliker, H., Miszkowicz, S., \& Stehling, W. 1991, Solar Phys., 133, 385

Benz, A. O., Magun, A., Stehling, W., \& Su, H. 1992, Solar Phys., 141, 335

Böhme, A., \& Krüger, A. 1982, Solar Phys., 76, 63

Canfield, R. C., et al. 1980, Solar Flares, Skylab Workshop II, ed. P. A. Sturrock (Boulder: Colorado Associated Univ. Press), 231
REFERENCES

Dennis, B. R., \& Zarro, D. M. 1993, Solar Phys., 146, 177

DeJager, C., \& DeJonge, G. 1978, Solar Phys., 58, 127

Doschek, G. A. 1990, ApJS, 73, 117

Doschek, G. A., et al. 1986, in Energetic Phenomena on the Sun: The Solar Maximum Mission Flare Workshop, ed. M. R. Kundu \& B. Woodgate, (NASA CP-2439), 4-1

Dulk, G. A. 1985, ARA\&A, 23, 169

Dulk, G. A., Sheridan, K. V., Smerd, S. F., \& Withbroe, G. L. 1977, Solar Phys., 52,349

Dulk, G. A., Steinberg, J. L., Hoang, S., \& Godman, M. V. 1987, A\&A, 173, 366

Fishman, G. J., et al. 1989, in The Burst and Transient Source Experiment (BATSE)-Scientific Objectives and Capabilities, GRO Science Workshop (Greenbelt: NASA/GSFC), 2

Fishman, G. J., Meegan, C. A., Wilson, R. B., Paciesas, W. S., \& Pendleton, G. N. 1992, in The Compton Observatory Science Workshop, ed. C. R. Shrader, N. Gehrels, \& B. R. Dennis NASA CP 3137, 26

Fokker, A. D. 1970, Solar Phys., 11, 92

Isliker, H., \& Benz, A. O. 1994, A\&AS, 104, 145

Lin, R. P., Levedahl, W. K., Lotko, W., Gurnett, D. A., \& Scarf, F. L. 1986, ApJ, 308, 954

Lin, R. P., Potter, D. W., Gurnett, D. A., \& Scarf, F. L. 1981, ApJ, 251, 364

Poquerusse, M. 1994, A\&A, 286, 611

Stähli, M., \& Benz, A. O. 1987, A\&A, 175, 271

Stewart, R. T. 1965, Australian J. Phys., 18, 67

Sturrock, P. A. 1973, in Symp. on High-Energy Phenomena in the Sun, ed. R. Ramaty \& R. G. Stone, (NASA/GSFC Rept. X-693-73-193), 3

Wild, J. P., Sheridan, K. V., \& Neylan, A. A. 1959, Australian J. Phys., 12, 369 